\title{
Pointed Hopf Algebras of Dimension 32
}

\author{
Matías Graña \\ Depto de Matemática, Pab. I, Ciudad Universitaria \\ (1428) Buenos Aires, Argentina \\ e-mail: matiasg@dm.uba.ar
}

\begin{abstract}
We give a complete classification of the 32-dimensional pointed Hopf algebras over an algebraically closed field $\mathbf{k}$ with char $\mathbf{k} \neq 2$. It turns out that there are infinite families of isomorphism classes of pointed Hopf algebras of dimension 32. In [AS1], [BDG] and [Ge] are given families of counterexamples for the tenth Kaplansky conjecture. Up to now, 32 is the lowest dimension where Kaplansky conjecture fails.
\end{abstract}

\section{INTRODUCTION}

In recent years there has been certain activity in classification problems of pointed Hopf algebras. In [AS1], [BDG], [Ge] independently the authors find infinite families of pointed Hopf algebras of dimension $p^{4}$ ( $p$ an odd prime number), solving thus by the negative the tenth Kaplansky conjecture. In [D] there is a classification of pointed Hopf algebras of dimension $p^{n}$ with coradical of dimension $p^{n-1}$.

In [AS1], [AS2], [AS3] there are classifications of pointed Hopf algebras of dimension $p^{3}, p^{4}$ and a large number of strong results towards the classification of pointed Hopf algebras with commutative coradical. Using the techniques of these articles, namely the "lifting procedure" described below, we classify here the pointed Hopf algebras of dimension 32 over an algebraically closed field of characteristic $\neq 2$. Related results can be found in [CDR] (the classification of the pointed Hopf algebras of dimension 16), in [Be] (classification of Ore extension Hopf algebras

This work was partially supported by CONICET, CONICOR, Secyt (UNC), Secyt (UBA) 
with coradical $\mathbf{k}\left(C_{2} \times C_{2}\right)$ and $\left.\mathbf{k} C_{4}\right)$, and in [G] (the description of the coradically graded pointed Hopf algebras of dimension $p^{5}$ ).

One of the key results is Corollary 2.14, where we compute the Nichols algebras $\mathfrak{B}(V)$ (see 1.2 for the definition of Nichols algebras), $V$ the Yetter-Drinfeld modules of dimension 2 over the groups of order $2^{j}, 1 \leq j \leq 4$.

Our main result is

Theorem 1.0.1. Let $A$ be a pointed Hopf algebra of dimension 32 over the algebraically closed field $\mathbf{k}$, char $\mathbf{k} \neq 2$. Let $\Gamma=G(A)$ be the group of group-likes of $A$.

If $\Gamma=C_{2}$ then $A$ is the algebra of section 3 .

If $\Gamma=C_{2} \times C_{2}$ then $A$ is one of the 6 algebras of 4.1.

If $\Gamma=C_{4}$ then $A$ is one of the 12 algebras of 4.2.

If $\Gamma=C_{2} \times C_{2} \times C_{2}$ then $A$ is one of the 6 algebras of 5.1 .

If $\Gamma=C_{2} \times C_{4}$ then $A$ is in the infinite family of algebras of 5.2.

If $\Gamma=C_{8}$ then $A$ is in the infinite family of algebras of 5.3 .

If $\Gamma=\mathbb{D}_{4}$ the dihedral group, then $A$ is one of the 7 algebras of 5.4 .

If $\Gamma=\mathbb{H}$ the quaternionic group, then $A$ is one of the 3 algebras of 5.5.

If $\Gamma=C_{2} \times C_{2} \times C_{2} \times C_{2}$ then $A$ is the algebra of 6.1 .

If $\Gamma=C_{2} \times C_{2} \times C_{4}$ then $A$ is one of the 7 algebras of 6.2.

If $\Gamma=C_{4} \times C_{4}$ then $A$ is one of the 4 algebras of 6.3 .

If $\Gamma=C_{2} \times C_{8}$ then $A$ is one of the 14 algebras of 6.4 .

If $\Gamma=C_{16}$ then $A$ is one of the 7 algebras of 6.5 .

If $\Gamma$ is one of the non abelian groups of order 16 then $A$ is one of the 13 algebras of 6.6.

If $\Gamma$ has order 32 then $A$ is a group algebra.

1.1. Some basic definitions and conventions. Throughout the article $\mathbf{k}$ shall be an algebraically closed field of characteristic $\neq 2$. The degrees for graded algebras shall be denoted by parentheses, i.e. $H(i)$ is the homogeneous component of degree $i$ of the graded algebra $H$. The symbols $\mathbf{i}, \xi$ shall denote fixed roots of unity of orders 4,8 respectively, such that $\mathbf{i}=\xi^{2}$.

Let $H$ be a Hopf algebra. We denote by ${ }_{H}^{H} \mathcal{Y} \mathcal{D}$ the category of (left-left) Yetter-Drinfeld modules over $H$, i.e. $M$ is an object of ${ }_{H}^{H} \mathcal{Y D}$ if it is a left $H$-module (with structure $h \otimes m \mapsto$ $h \rightarrow m$ ), a left $H$-comodule (with structure $\delta(m)=m_{(-1)} \otimes m_{(0)}$ ) and such that

$$
\delta(h \rightarrow m)=h_{(1)} m_{(-1)} \mathcal{S}\left(h_{(3)}\right) \otimes h_{(2)} m_{(0)} .
$$

We denote by $c$ the braiding in ${ }_{H}^{H} \mathcal{Y} \mathcal{D}$, which is given by

$$
c=c_{M, N}: M \otimes N \rightarrow N \otimes M, \quad m \otimes n \mapsto m_{(-1)} \rightarrow n \otimes m_{(0)} .
$$

When $H$ is clear from the context, we shall call a module in ${ }_{H}^{H} \mathcal{Y D}$ a YD-module. Unless explicitly stated, the letter $V$ shall denote a YD-module. 
For $\Gamma$ a group and $g \in \Gamma$ we denote by $\Gamma_{g}$ the commutator subgroup

$$
\Gamma_{g}=\{h \in \Gamma \mid h g=g h\} .
$$

It is not difficult to prove (see for instance $[\mathrm{AG}]$ ) that if $H=\mathbf{k} \Gamma$ and $|\Gamma| \neq 0$ in $\mathbf{k}$ then ${ }_{H}^{H} \mathcal{Y} \mathcal{D}$ is a semisimple category and the irreducible objects are given as follows: let $g \in \Gamma$ and $\rho: \Gamma_{g} \rightarrow \operatorname{Aut}(W) \in \widehat{\Gamma_{g}}$ an irreducible representation. Then the induced representation

$$
M(g, \rho)=\operatorname{Ind}_{\Gamma_{g}}^{\Gamma} W=\mathbf{k} \Gamma \otimes_{\mathbf{k} \Gamma_{g}} W
$$

with the usual module structure and the comodule structure given by

$$
\delta(h \otimes w)=h g h^{-1} \otimes(h \otimes w) \in \mathbf{k} \Gamma \otimes M(g, \rho)
$$

is an irreducible YD-module. Furthermore, let $\left\{g_{1}, \ldots, g_{n}\right\}$ be a subset of $\Gamma$ consisting of one element for each conjugacy class, and let $\left\{\rho_{i}^{1}, \ldots, \rho_{i}^{m_{i}}\right\}$ be the irreducible representations of $\Gamma_{g_{i}}$. Then the objects $M\left(g_{i}, \rho_{i}^{j}\right)\left(1 \leq i \leq n, 1 \leq j \leq m_{i}\right)$ gives a full collection of non-isomorphic irreducible objects of $\mathbf{k}_{\mathbf{k} \Gamma} \mathcal{Y} \mathcal{D}$.

We extend the definition of $M(g, \rho)$ to the case in which $\rho$ is not irreducible. Observe that $\operatorname{dim} M(g, \rho)=\operatorname{deg} \rho \times\left[\Gamma: \Gamma_{g}\right]$. Hence, if $\Gamma$ is abelian, the irreducible modules of $\mathrm{k} \Gamma \mathcal{Y} \mathcal{D}$ are 1-dimensional. In this case, let $V=\oplus_{i} M\left(h_{i}, \chi_{i}\right)$ where $h_{i} \in \Gamma$ and $\chi_{i} \in \hat{\Gamma}$, and denote by $x_{i}$ a generator of $M\left(h_{i}, \chi_{i}\right)$. By the definition of the braiding,

$$
c\left(x_{i} \otimes x_{j}\right)=\chi_{j}\left(h_{i}\right) x_{j} \otimes x_{i} .
$$

Let $q \in \mathbf{k}$. For $n \geq m \in \mathbb{N}$, we use the standard notation

$$
(n)_{q}=\sum_{i=0}^{n-1} q^{i}, \quad(n)_{q}^{!}=\prod_{i=1}^{n}(i)_{q}, \quad\left(\begin{array}{c}
n \\
m
\end{array}\right)_{q}=\frac{(n)_{q}^{!}}{(m)_{q}^{!}(n-m)_{q}^{!}} .
$$

Let $H$ be a graded Hopf algebra, $H=\oplus_{i} H(i)$. Let $\pi: H \rightarrow H(0)$ and $\iota: H(0) \rightarrow H$ be respectively the canonical projection and the canonical inclusion. Then

$$
R=H^{\mathrm{co} \pi}=H^{\mathrm{co} H(0)}=\{x \in H \mid(\mathrm{id} \otimes \pi) \Delta(x)=x \otimes 1\}
$$

is a braided Hopf algebra in ${ }_{H(0)}^{H(0)} \mathcal{Y D}$. Furthermore, it is graded: $R=\oplus_{i} R(i)$, with $R(i)=$ $R \cap H(i)$. The algebra $H$ can be reconstructed as a bosonization, or biproduct (see e.g. [AG])

$$
H=R \# H(0) \text {. }
$$

A braided Hopf algebra $R$ in ${ }_{K}^{K} \mathcal{Y} \mathcal{D}$ ( $K$ a Hopf algebra) is called Nichols algebra if

$$
\begin{aligned}
& R=\oplus_{i} R(i) \text { is a graded braided Hopf algebra, } \\
& R(0)=\mathbf{k} 1 \\
& \mathcal{P}(R)=R(1) \\
& R(1) \text { generates } R
\end{aligned}
$$


where $\mathcal{P}(R)$ stands for the space of primitive elements of $R$. It is not known whether in characteristic 0 and for $R$ finite dimensional the condition (1.1.5), resp. (1.1.4), is superfluous, though there are some results in this direction. For instance, in [AS1, Thm. 3.2] is proved that if $\operatorname{dim} R(1)=1$ ( $R$ finite dimensional and char $\mathbf{k}=0)$ then $((1.1 .2)-(1.1 .4)) \Rightarrow(1.1 .5)$. A Nichols algebra $R$ is uniquely determined by its primitive elements $\mathcal{P}(R)$. We use hence the notation $R=\mathfrak{B}(V)$ for the Nichols algebra $R$ with $R(1)=V$, whenever $V \in{ }_{K}^{K} \mathcal{Y} \mathcal{D}$. We specify sometimes the graduation of $\mathfrak{B}(V)$ by

$$
\mathfrak{B}(V)=\oplus_{i} \mathfrak{B}^{i}(V) .
$$

The construction $V \mapsto \mathfrak{B}(V)$ can be made with the so called quantum-shuffles (see for instance $[\mathrm{AG}]$ ), though this construction does not depend on the braided category ${ }_{K}^{K} \mathcal{Y} \mathcal{D}$, but only on the pair $(V, c)$. The quantum-shuffles construction applied to any pair $(V, c)(c \in$ $\operatorname{End}(V \otimes V)$ a solution of the braid equation), the result has been called Quantum Shuffle Algebra in [Ros]. Nichols algebras have some particularly useful properties which distinguish them from this general concept of Quantum Shuffle Algebras, e.g. the kind of Poincaré duality stated in 2.7 .

We shall use the following notation for finite abelian groups: let $\Gamma=C_{n_{1}} \times \cdots \times C_{n_{t}}$. We denote by $g_{j}$ a generator of the $j$-th factor, i.e. $\Gamma$ can be presented by generators $g_{j}$ and relations $g_{j}^{n_{j}}=\left[g_{j}, g_{k}\right]=1$. Furthermore, let $\alpha_{j}=-1, \mathbf{i}, \xi, \sqrt{\xi}$ depending whether $n_{j}=2,4,8,16$ (recall that $\left.\xi^{2}=\mathbf{i}, \mathbf{i}^{2}=-1\right)$. We denote by $\hat{g}_{i}$ the character of $\Gamma$ given by $\hat{g}_{i}\left(g_{j}\right)=\alpha_{i}^{\delta_{i j}}$. Then $\hat{\Gamma} \simeq \Gamma$ with generators $\hat{g}_{i}, i=1, \ldots, t$.

If $x_{1}, \ldots, x_{r}$ are vectors in a space $V$, we denote by $\left(x_{1}, \ldots, x_{r}\right)$ the linear span of them. If $g_{1}, \ldots, g_{r}$ are elements in $\Gamma$, we denote by $\left(g_{1}, \ldots, g_{r}\right)$ the subgroup generated by them.

If $V \in{ }_{\mathbf{k} \Gamma}^{\mathbf{k} \Gamma} \mathcal{Y} \mathcal{D}$ and $\Gamma$ is abelian then $V=\oplus_{i=1}^{\theta} M\left(h_{i}, \chi_{i}\right)$. We denote by $x_{i}$ a generator of $M\left(h_{i}, \chi_{i}\right)$, whence $V=\left(x_{1}, \ldots, x_{\theta}\right)$ and $c$ is given by the matrix $\left(b_{i j}\right)$, with $b_{i j}=\chi_{j}\left(h_{i}\right)$.

1.2. Lifting Procedure. Let $A$ be a finite dimensional pointed Hopf algebra. Let $A_{0} \subset A_{1} \subset$ ... be its coradical filtration, and consider

$$
H=\oplus_{i} H(i)=\operatorname{gr} A=\oplus_{i}\left(A_{i} / A_{i-1}\right) \quad\left(A_{-1}=0\right) .
$$

It is a coradically graded Hopf algebra, i.e. $H_{n}=\oplus_{i=0}^{n} H(i)$. Take $R=H^{\mathrm{co} H(0)}$. This is a coradically graded Hopf algebra in $\mathrm{k} \Gamma_{\mathrm{k}} \mathcal{Y} \mathcal{D}$, where $\Gamma=G(A)$ is the group of group-likes of $A$ (and hence $H(0)=\mathrm{k} \Gamma$ ). Notice that the order of $\Gamma$ divides the dimension of $A$, by virtue of the Nichols-Zoeller theorem. Furthermore, $R(0)=\mathbf{k} 1$, from where $R$ satisfies (1.1.2), (1.1.3) and (1.1.4). If moreover $R$ satisfies (1.1.5) then it is a Nichols algebra, and hence $R=\mathfrak{B}(R(1))$. Since $R$ is an invariant of $A$, we shall consider its "infinitesimal part" defined by

$$
V=V(A)=R(1)
$$

and call $\operatorname{dim} V$ the rank of $A$. It is clear that the $\operatorname{rank}$ of $A$ is equal to $\operatorname{dim} A_{1} / \operatorname{dim} A_{0}-1$. 
The lifting procedure goes the other way: let $n \in \mathbb{N}$ be fixed. We want to classify the $n$ dimensional pointed Hopf algebras over $\mathbf{k}$. We can proceed as follows: for each group $\Gamma$ such that $|\Gamma|$ divides $n$,

1. Determine the modules $V \in \underset{\mathbf{k} \Gamma}{\mathbf{k} \Gamma} \mathcal{Y D}$ such that $\operatorname{dim} \mathfrak{B}(V) \leq \frac{n}{|\Gamma|}$.

2. Classify the isomorphism classes of the bosonizations $\mathfrak{B}(V) \# \mathbf{k} \Gamma$, where $V$ runs over the modules in step 1. Note that two bosonizations $\mathfrak{B}(V) \# \mathbf{k} \Gamma$ and $\mathfrak{B}(V)^{\prime} \# \mathbf{k} \Gamma$ may be isomorphic even though $\mathfrak{B}(V)$ and $\mathfrak{B}(V)^{\prime}$ (thus, $V$ and $V^{\prime}$ ) are not isomorphic (see 2.16).

3. For each isomorphism class $\mathfrak{B}(V) \# \mathbf{k} \Gamma$ in step 2, classify all the liftings, i.e. all the pointed Hopf algebras (up to isomorphism) such that gr $A=\mathfrak{B}(V) \# \mathbf{k} \Gamma$.

4. Prove, if possible, that these are all the $n$-dimensional pointed Hopf algebras. Equivalently, show that a graded braided Hopf algebra $R$ in ${ }_{\mathrm{k} \Gamma}^{\mathrm{k} \Gamma} \mathcal{Y} \mathcal{D}$ such that $\operatorname{dim} R=n /|\Gamma|$ and satisfying (1.1.2)-(1.1.4), also satisfies (1.1.5) (for this purpose it is useful to classify those $V$ in step 1 such that $\operatorname{dim} \mathfrak{B}(V)<n /|\Gamma|)$.

1.3. Contents. The article is organized as follows: in section 2 we compute the Nichols algebras that appear applying the procedure above for 32-dimensional pointed Hopf algebras.

From 3 to 6.6 we follow the steps 1, 2 and 3 of the procedure for each of the groups of order $2^{m}, m \leq 4$. In section 7 we prove the statement in step 4 of the procedure.

This work is part of the doctoral thesis of the author, who thanks N. Andruskiewitsch for his excellent guidance. I thank also H.-J. Schneider and M. Lamas for many valuable conversations.

Our main references on Hopf algebras are $[\mathrm{S}]$ and $[\mathrm{M}]$. We refer to the survey article [AG] for definitions and properties of braided Hopf algebras, Nichols algebras (called there TOBAs) and for further references on the subject.

\section{The Nichols Algebras We Shall NeED}

For future reference, we give two families of examples of Nichols algebras. The first one corresponds to Taft algebras, and the second one to the more general case of Quantum Linear Spaces (QLS for brevity).

Example 2.1. Let $V$ be 1-dimensional and let $x$ be a generator of $V$. Suppose $c(x \otimes x)=q x \otimes x$, where $q \in \mathbf{k}^{\times}$. If $q$ is a root of unity of order $n>1$, or if $q=1$ and char $\mathbf{k}=n>0$, then $\mathfrak{B}(V)=\mathbf{k}[x] /\left(x^{n}\right)$ as algebra (and hence $\operatorname{dim} \mathfrak{B}(V)=n$ ). If $q$ is not a root of unity (which can happen for instance for $V \in \mathbf{k} \mathbb{Z} \mathbb{Z} \mathcal{Y})$, or if $q=1$ and char $\mathbf{k}=0$, then $\mathfrak{B}(V)=\mathbf{k}[x]$ and hence it is infinite dimensional. The comultiplication in both cases is given by $\Delta\left(x^{d}\right)=\sum_{i=0}^{d}\left(\begin{array}{l}d \\ i\end{array}\right)_{q} x^{i} \otimes x^{d-i}$.

Remark 2.2. The above example shows the following useful result: let $H$ be a finite dimensional Hopf algebra and $R \in{ }_{H}^{H} \mathcal{Y} \mathcal{D}$ a braided Hopf algebra of dimension $N$. Suppose char $\mathbf{k}$ does not divide $N$. If $x \in \mathcal{P}(R)$ is such that $\mathbf{k} x$ is a sub-YD-module of $R$ and $c(x \otimes x)=q x \otimes x$, where $q \in \mathbf{k}^{\times}$, then $q \neq 1$. 
Proof. Suppose $q=1$. If char $\mathbf{k}=0$ then $R$ would be infinite dimensional, if char $\mathbf{k}=p>0$ then $x$ would generate an algebra of dimension a power of $p$, but then, taking the bosonization of both algebras, $p$ would divide $N$.

Definition 2.3. Let $q \in \mathbf{k}^{\times}$. We define $N(q)$ by

$$
N(q)= \begin{cases}\text { order of } q & \text { if } q \neq 1 \text { and is a root of unity, } \\ \infty & \text { if } q \text { is not a root of unity, } \\ \infty & \text { if } q=1 \text { and char } \mathbf{k}=0 \\ \text { char } \mathbf{k} & \text { if } q=1 \text { and char } \mathbf{k}>0\end{cases}
$$

Definition 2.4. Let $V \in{ }_{H}^{H} \mathcal{Y D}$ be of dimension $\theta$. We shall say that $V$ (or $c$ ) has a matrix if there exists a basis $\left\{x_{1}, \ldots, x_{\theta}\right\}$ of $V$ and a matrix $\left(b_{i j}\right) \in M_{\theta}\left(\mathbf{k}^{\times}\right)$such that the braiding $c$ is given by

$$
c\left(x_{i} \otimes x_{j}\right)=b_{i j} x_{j} \otimes x_{i} .
$$

When we want to specify the basis, we say that $V$ has a matrix $\left(b_{i j}\right)$ in the basis $\left\{x_{1}, \ldots, x_{\theta}\right\}$. If $H=\mathbf{k} \Gamma$ and $\Gamma$ is abelian, then every finite dimensional module has a matrix (see (1.1.1)).

Let $V \in \underset{\mathbf{k} \Gamma}{\mathbf{k}} \mathcal{Y} \mathcal{D}$ be finite dimensional. We shall say that $V$ (or c) comes from the abelian case if there exists an invariant abelian subgroup $\Gamma^{\prime} \subset \Gamma$ such that the image of $\delta: V \rightarrow \mathrm{k} \Gamma \otimes V$ is included in $\mathrm{k} \Gamma^{\prime} \otimes V$. In this case, $V$ can be considered as a module in ${ }_{\mathbf{k} \Gamma^{\prime}}^{\mathrm{k}} \mathcal{Y} \mathcal{D}$ (thus, it has a matrix) and the bosonization $\mathfrak{B}(V) \# \mathbf{k} \Gamma$ can be reconstructed as an extension of $\Gamma / \Gamma^{\prime}$ by $\mathfrak{B}(V) \# \mathbf{k} \Gamma^{\prime}$. We note that the condition on $\Gamma^{\prime}$ to be invariant can be dropped: it is not difficult to see that if there exists $\Gamma^{\prime}$ abelian such that the image of $\delta$ lies in $\mathrm{k} \Gamma^{\prime} \otimes V$, then there exists $\Gamma^{\prime \prime} \subset \Gamma^{\prime}$ invariant (and clearly abelian) such that the image of $\delta$ lies in $\mathrm{k} \Gamma^{\prime \prime} \otimes V$.

Example 2.5. Let $V \in{ }_{H}^{H} \mathcal{Y D}$ be of dimension $\theta$, and suppose $V$ has a matrix $\left(b_{i j}\right)$ in the basis $\left\{x_{1}, \ldots, x_{\theta}\right\}$. Let $N_{i}=N\left(b_{i i}\right)$, see 2.3. Then it can be proved (see [AS1, Prop. 3.5]) that

$$
\operatorname{dim} \mathfrak{B}(V) \geq \prod_{i=1}^{n} N_{i}
$$

Furthermore, if $N_{i}<\infty$ for all $i$, then the equality holds if and only if

$$
b_{i j} b_{j i}=1 \text { for all } i \neq j .
$$

In this case, a PBW basis of $\mathfrak{B}(V)$ is given by

$$
\left\{x_{1}^{n_{1}} \cdots x_{\theta}^{n_{\theta}} \mid 0 \leq n_{i}<N_{i}\right\}
$$

and the multiplication is given by the following relations:

$$
x_{i}^{N_{i}}=0, \quad x_{j} x_{i}=b_{j i} x_{i} x_{j} \text { for } i<j .
$$

Hence, as an algebra, $\mathfrak{B}(V)$ is a quantum linear space. Let now $H=\mathbf{k} \Gamma$. In order to agree with the notation of [AS1], we shall say that $\mathfrak{B}(V)$ is a Quantum Linear Space (or QLS) if $V$ 
satisfies (2.6) and the lines $\mathbf{k} x_{i}$ are submodules of $V$ in ${ }_{\mathbf{k} \Gamma}^{\mathbf{k} \Gamma} \mathcal{Y} \mathcal{D}$. If $V$ can be considered as a module in $\mathrm{k}_{\mathrm{k}} \Gamma^{\prime} \mathcal{Y} \mathcal{D}$ for certain subgroup $\Gamma^{\prime} \subset \Gamma$ (that is, if the image of the coaction $\delta$ lies in $\left.\Gamma^{\prime} \otimes V\right)$ and it is a QLS in $\underset{\mathrm{k} \Gamma^{\prime}}{\mathrm{k} \Gamma^{\prime}} \mathcal{Y D}$ then we say that it is a QLS over $\Gamma^{\prime}$.

We shall use an important result about Nichols algebras due to Nichols:

Proposition 2.7. Let $R=\oplus_{i=0}^{N} R(i)$ be a Nichols algebra (in some braided rigid abelian category). Suppose that $R(N) \neq 0$. Then

$$
\operatorname{dim} R(i)=\operatorname{dim} R(N-i) \quad \forall i=0, \ldots, N .
$$

Proof. See for instance [AG, Pr. 3.2.2].

Lemma 2.8. Let $A$ be a $2^{n}$-dimensional pointed Hopf algebra with $A_{0}=\mathbf{k} \Gamma$. Suppose $|\Gamma| \geq$ $2^{n-2}$ and let $V=V(A) \in{ }_{\mathbf{k} \Gamma}^{\mathbf{k} \Gamma \mathcal{D}}$ be the module defined by (1.2.1). Then $\operatorname{dim} V \leq 2$.

Proof. Since $2^{n}=|\Gamma| \times \operatorname{dim} \mathfrak{B}(V)$ we have $\operatorname{dim} \mathfrak{B}(V) \leq 4 ;$ but if $\operatorname{dim} V \geq 3$, then $\operatorname{dim} \mathfrak{B}(V) \geq 5$ by 2.7 .

In $[\mathrm{N}]$ the author introduces derivations for Nichols algebras, which turns out to be a useful tool for computing bases (and hence their dimensions).

Definition 2.9. Let $V \in{ }_{H}^{H} \mathcal{Y D}$. For $i+j=n$, we denote by

$$
\Delta_{i, j}: \mathfrak{B}^{n}(V) \rightarrow \mathfrak{B}^{i}(V) \otimes \mathfrak{B}^{j}(V)
$$

the $(i, j)$-component of the comultiplication of $\mathfrak{B}(V)$. It is proved in [Sch] (or see [AG, Def. 3.2.10]) that $\Delta_{i, j}$ is injective $\forall i, j$. Let $\left\{x_{1}, \ldots, x_{\theta}\right\}$ be a basis of $V$ and let $\left\{x_{1}^{*}, \ldots, x_{\theta}^{*}\right\}$ be its dual basis. We denote by $\partial_{x_{i}}$ the differential operator on $\mathfrak{B}(V)$ given by

$$
\partial_{x_{i}}(z)=\left(\mathrm{id} \otimes x_{i}^{*}\right) \Delta_{n-1,1}(z), \quad \text { if } z \in \mathfrak{B}^{n}(V), n>0, \quad \text { and } \partial_{x_{i}}(1)=0 .
$$

By the injectivity of $\Delta_{i, j}$ it is clear that for $z \in \mathfrak{B}^{n}(V)(n>0)$ we have $z=0$ if and only if $\partial_{x_{i}}(z)=0$ for all $i=1, \ldots, \theta$. Suppose now that $V \in \underset{\mathbf{k} \Gamma}{\mathrm{k} \Gamma \mathcal{D}}$ and some $x_{i}^{*}$ vanishes on the homogeneous components of degree $\neq g_{i}$ for some $g_{i} \in \Gamma$ (that is, $x_{i}^{*}(y)=0$ if $\delta(y)=h \otimes y$ and $\left.h \neq g_{i}\right)$. Then it is easy to see that $\partial_{x_{i}}$ verifies the Leibniz rule

$$
\partial_{x_{i}}\left(z_{1} z_{2}\right)=\partial_{x_{i}}\left(z_{1}\right)\left(g_{i} \rightarrow z_{2}\right)+z_{1} \partial_{x_{i}}\left(z_{2}\right) .
$$

Since usually we shall take bases $\left\{x_{1}, \ldots, x_{\theta}\right\}$ of homogeneous elements (i.e. $\delta\left(x_{i}\right)=g_{i} \otimes x_{i}$ for some $\left.g_{i} \in \Gamma\right)$, the previous condition will hold for all $x_{i}^{*}$.

Definition 2.10. Let $A$ be a Hopf algebra. We shall denote by Ad the adjoint action

$$
\operatorname{Ad}_{x}(y)=x_{(1)} y \mathcal{S}\left(x_{(2)}\right)
$$


Let $B$ be a braided Hopf algebra with multiplication $\mu$, let $c \in \operatorname{End}(B \otimes B)$ be the braiding. We shall denote by Ad the braided adjoint action, given by

$$
\operatorname{Ad}_{x}(y)=\mu(\mu \otimes \mathcal{S})(\mathrm{id} \otimes c)(\Delta \otimes \mathrm{id})(x \otimes y)
$$

Note that if $x \in \mathcal{P}(B)$ then the adjoint is nothing but $\operatorname{Ad}_{x}(y)=\mu(\mathrm{id}-c)(x \otimes y)$. Since in the cases we are interested in the endomorphism $c$ is given by the context, we do not make a reference to $c$ in the notation.

We need the following relation between both adjoint actions: let $H$ be a graded Hopf algebra, $\pi: H \rightarrow H(0)$ the canonical projection and $B \in_{H(0)}^{H(0)} \mathcal{Y D}$ the braided Hopf algebra of coinvariants (then $H \simeq B \# H(0)$ ). Let $x \in B(1)$ (it is a primitive element). Then it is straightforward to see that

$$
\operatorname{Ad}_{(b \# 1)}(c \# 1)=\left(\operatorname{Ad}_{b}(c)\right) \# 1 \quad \forall c \in B,
$$

where the left Ad is the classical one, while the right Ad is the braided one.

We shall compute now the Nichols algebras appearing in next sections.

Proposition 2.11. Let $V \in{ }_{H}^{H} \mathcal{Y D}$ with basis $\{x, y\}$. Suppose that c has a matrix $\left(b_{i j}\right)$ in this basis, and suppose that $b_{12} b_{21} \neq 1, b_{11} \neq 1, b_{22} \neq 1$. Let $z=\operatorname{Ad}_{x}(y)=x y-b_{12} y x$. Let $N_{i}=N\left(b_{i i}\right)$ (see 2.3) and suppose that $N_{i}<\infty$ (i.e., that $b_{i i}$ is a root of unity, or $b_{i i}=1$ and char $\mathbf{k}>0)$. Let $q=b_{11} b_{12} b_{21} b_{22}$.

1. There exists $n_{12}$ (respectively $\left.n_{21}\right)$ such that $\left(\operatorname{Ad}_{x}\right)^{n_{12}}(y)=0$ (respectively $\left.\left(\operatorname{Ad}_{y}\right)^{n_{21}}(x)=0\right)$. Let $n_{12}$ (resp. $n_{21}$ ) be the least positive integer with such property. Then $n_{12} \leq N_{1}$ and $n_{21} \leq N_{2}$. Suppose that there exists $m_{12}$ (resp. $m_{21}$ ) such that $b_{12} b_{21} b_{11}^{m_{12}}=1$ (resp. $b_{12} b_{21} b_{22}^{m_{21}}=1$ ) and let $m_{12}$ (resp. $\left.m_{21}\right)$ be the least non negative integer with such property. Then $n_{12}=m_{12}+1$ (resp. $\left.n_{21}=m_{21}+1\right)$.

2. If $q=1$ and char $\mathbf{k}=0$ then $z^{n} \neq 0 \forall n$ and then $\mathfrak{B}(V)$ is infinite dimensional. If $q=1$ and char $\mathbf{k} \neq 0$ then $\operatorname{dim} \mathfrak{B}(V) \geq N_{1} N_{2}$ char $\mathbf{k}$.

3. Suppose $b_{11}=b_{22}=-1$ and let $N=N(q)$. Then $\mathfrak{B}(V)$ has dimension $4 N$, with a $P B W$ basis $\left\{x^{a} y^{b} z^{c} \mid 0 \leq a, b<2,0 \leq c<N\right\}$.

Proof. 1. We compute inductively the derivatives of $\left(\operatorname{Ad}_{x}\right)^{n}(y)$ (see 2.9):

$$
\partial_{x}\left(\left(\operatorname{Ad}_{x}\right)^{1}(y)\right)=\partial_{x}\left(x y-b_{12} y x\right)=0 .
$$

Suppose that $\partial_{x}\left(\left(\operatorname{Ad}_{x}\right)^{n}(y)\right)=0$, then

$$
\begin{aligned}
\partial_{x}\left(\left(\operatorname{Ad}_{x}\right)^{n+1}(y)\right) & =\partial_{x}\left(x\left(\operatorname{Ad}_{x}\right)^{n}(y)-b_{11}^{n} b_{12}\left(\operatorname{Ad}_{x}\right)^{n}(y) x\right) \\
& =b_{11}^{n} b_{12}\left(\operatorname{Ad}_{x}\right)^{n}(y)-b_{11}^{n} b_{12}\left(\operatorname{Ad}_{x}\right)^{n}(y)=0 \\
\partial_{y}\left(\left(\operatorname{Ad}_{x}\right)^{1}(y)\right) & =\partial_{y}\left(x y-b_{12} y x\right)=\left(1-b_{12} b_{21}\right) x .
\end{aligned}
$$


Let $\sigma_{n}=\left(1-b_{12} b_{21} b_{11}^{n}\right)$. Suppose that $\partial_{y}\left(\left(\operatorname{Ad}_{x}\right)^{n}(y)\right)=\sigma_{0} \sigma_{1} \cdots \sigma_{n-1} x^{n}$. Then

$$
\begin{aligned}
\partial_{y}\left(\left(\operatorname{Ad}_{x}\right)^{n+1}(y)\right) & =\partial_{y}\left(x\left(\operatorname{Ad}_{x}\right)^{n}(y)-b_{11}^{n} b_{12}\left(\operatorname{Ad}_{x}\right)^{n}(y) x\right) \\
& =\sigma_{0} \cdots \sigma_{n-1} x^{n+1}-b_{11}^{n} b_{12} \sigma_{0} \cdots \sigma_{n-1} b_{21} x^{n+1} \\
& =\sigma_{0} \cdots \sigma_{n} x^{n+1} .
\end{aligned}
$$

Thus, since $x^{N_{1}}=0$, we have

$$
\partial_{x}\left(\left(\operatorname{Ad}_{x}\right)^{N_{1}}(y)\right)=\partial_{y}\left(\left(\operatorname{Ad}_{x}\right)^{N_{1}}(y)\right)=0
$$

and hence $\left(\operatorname{Ad}_{x}\right)^{N_{1}}(y)=0$, which proves that $n_{12}$ exists and $n_{12} \leq N_{1}$. Furthermore, if $b_{12} b_{21} b_{11}^{m_{12}}=1$, we see that

$$
\partial_{x}\left(\left(\operatorname{Ad}_{x}\right)^{m_{12}+1}(y)\right)=\partial_{y}\left(\left(\operatorname{Ad}_{x}\right)^{m_{12}+1}(y)\right)=0
$$

whence $n_{12} \leq m_{12}+1$ (notice that if $m_{12}$ exists, then $m_{12}<N_{1}$ ). Moreover, the elements $x^{i}$ do not vanish for $i<N_{1}$, whence if $m_{12}$ exists then $\partial_{y}\left(\left(\operatorname{Ad}_{x}\right)^{m_{12}}(y)\right) \neq 0$, from where $n_{12}>m_{12}$ and thus $n_{12}$ is exactly $m_{12}+1$.

2. We compute the derivatives of $z^{n}$. We do it for a general $q=b_{11} b_{12} b_{21} b_{22}$ (keep the notation $\left.\sigma_{n}=\left(1-b_{12} b_{21} b_{11}^{n}\right)\right)$.

$$
\begin{gathered}
\partial_{x}(z)=\partial_{x}\left(\operatorname{Ad}_{x} y\right)=0 \\
\partial_{y}(z)=\partial_{y}\left(\operatorname{Ad}_{x} y\right)=\sigma_{0} x \\
\partial_{x} \partial_{y}(z)=\partial_{x}\left(\sigma_{0} x\right)=\sigma_{0} \\
\partial_{y}\left(z^{n}\right)=\sum_{i=0}^{n-1}\left(b_{21} b_{22}\right)^{i} \sigma_{0} z^{n-1-i} x z^{i}, \\
\partial_{x} \partial_{y}\left(z^{n}\right)=\sum_{i=0}^{n-1}\left(b_{21} b_{22}\right)^{i}\left(b_{11} b_{12}\right)^{i} \sigma_{0} z^{n-1} \\
=(n)_{q} \sigma_{0} z^{n-1} .
\end{gathered}
$$

Let now $q=1$. If char $\mathbf{k}=0$, we see inductively that $z^{n} \neq 0 \forall n$. Furthermore, since $z^{n} \in \mathfrak{B}^{2 n}(V)$, the set $\left\{z^{n} \mid n \geq 0\right\}$ is linearly independent, and thus $\mathfrak{B}(V)$ is infinite dimensional. If char $\mathbf{k}>0$, we see inductively that $z^{n} \neq 0 \forall n<$ char $\mathbf{k}$. Furthermore, with the same computations as in 3 below, we see that the set

$$
\left\{x^{a} y^{b} z^{c} \mid 0 \leq a<N_{1}, 0 \leq b<N_{2}, 0 \leq c<\operatorname{char} \mathbf{k}\right\}
$$

is linearly independent, whence $\operatorname{dim} \mathfrak{B}(V) \geq N_{1} N_{2}$ char $\mathbf{k}$. 
3. By the same computations than in 2, we have that the set $\left\{z^{i} \mid 0 \leq i<N\right\}$ is linearly independent. We first prove that $z^{N}=0$ :

$$
\begin{aligned}
& \partial_{x}\left(z^{N}\right)=0 \quad(\text { as in } 2) \\
& \partial_{y}\left(z^{N}\right)=\sum_{i=0}^{N-1}\left(b_{21} b_{22}\right)^{i} \sigma_{0} z^{N-1-i} x z^{i},
\end{aligned}
$$

but this element vanishes in $\mathfrak{B}(V)$, since

$$
\begin{aligned}
\partial_{x}\left(\partial_{y} z^{N}\right)= & (N)_{q} \sigma_{0} z^{N-1}=0 \quad(\text { as in } 2) \\
\partial_{y}\left(\partial_{y} z^{N}\right)= & \sum_{i=0}^{N-1}\left(b_{21} b_{22}\right)^{i} \sigma_{0}\left[\sum_{j=0}^{N-2-i}\left(b_{21} b_{22}\right)^{j+i} b_{21} z^{N-2-i-j} x z^{j} x z^{i}\right. \\
& \left.\quad+\sum_{j=0}^{i-1}\left(b_{21} b_{22}\right)^{j} z^{N-1-i} x z^{i-j-1} x z^{j}\right] \\
= & \sum_{k+l \leq N-2}\left(b_{21} b_{22}\right)^{k+2 l} \sigma_{0} b_{21}\left(1+b_{22}\right) z^{N-2-k-l} x z^{k} x z^{l} \\
= & 0\left(\text { since } b_{22}=-1\right) .
\end{aligned}
$$

Now, since $b_{11}=b_{22}=-1$, we have $x^{2}=y^{2}=0$. Furthermore,

$$
\begin{aligned}
& y x=b_{12}^{-1} x y-b_{12}^{-1} z, \\
& z x=\left(x y-b_{12} y x\right) x=x y x=-b_{12}^{-1} x\left(x y-b_{12} y x\right)=-b_{12}^{-1} x z, \\
& z y=\left(x y-b_{12} y x\right) y=-b_{12} y x y=-b_{12} y\left(x y-b_{12} y x\right)=-b_{12} y z .
\end{aligned}
$$

Thus the set $\left\{x^{a} y^{b} z^{c} \mid 0 \leq a, b<2,0 \leq c<N\right\}$ generates $\mathfrak{B}(V)$ as a vector space. Let $\alpha_{b, c} \in \mathbf{k}$ such that

$$
\sum_{\substack{0 \leq b<2 \\ 0 \leq c<N}} \alpha_{b, c} y^{b} z^{c}=0 .
$$

We compute

$$
\begin{aligned}
\partial_{y}\left(y z^{c}\right) & =\left(b_{21} b_{22}\right)^{c} z^{c}+y \partial_{y} z^{c}, \\
\partial_{x} \partial_{y}(y) & =0, \\
\partial_{x} \partial_{y}\left(y z^{c}\right) & =(c)_{q} \sigma_{0} y z^{c-1} \quad(c>0) .
\end{aligned}
$$

Applying $\left(\partial_{x} \partial_{y}\right)^{N-1}$, we see that $\alpha_{0, N-1}=\alpha_{1, N-1}=0$. Inductively, we see that $\alpha_{b, c}=$ $0 \forall b, c$. In the same vein, let $\alpha_{a, b, c} \in \mathbf{k}$ such that

$$
\sum_{\substack{0 \leq a, b<2 \\ 0 \leq c<N}} \alpha_{a, b, c} x^{a} y^{b} z^{c}=0 .
$$

We compute

$$
\partial_{x}\left(x y^{b} z^{c}\right)=b_{11}^{c} b_{22}^{b+c} y^{b} z^{c}
$$


from where we see that $\alpha_{1, b, c}=0 \forall b, c$, and thus $\alpha_{a, b, c}=0 \forall a, b, c$, which proves the statement.

Remark 2.12. It can be proved in general that if $V$ has a matrix $\left(b_{i j}\right)$ in a basis $\left\{x_{1}, \ldots, x_{\theta}\right\}$, then the operator $\left(\partial_{x_{i}}\right)^{N\left(b_{i i}\right)}$ vanishes. Furthermore, the duality of [AG, Prop. 3.2.30] translates products into compositions.

Definition 2.13. Let $V$ be a $\theta$-dimensional YD module, and suppose $V$ has a matrix $\left(b_{i j}\right)$. According to $[\mathrm{AS} 2]$, we say that $V$ is of Cartan type (or CT) if there exists a matrix $\left(a_{i j}\right) \in$ $M_{\theta}(\mathbb{Z})$ such that

$$
b_{i j} b_{j i}=b_{i i}^{a_{i j}} \quad \forall i, j .
$$

If we choose $a_{i i}=2$ and -order of $b_{i i}<a_{i j} \leq 0$ for $i \neq j$, then $\left(a_{i j}\right)$ is a generalized Cartan matrix. We transfer to $V$ the terminology on $\left(a_{i j}\right)$.

\section{Corollary 2.14.}

1. Let $V \in{ }_{H}^{H} \mathcal{Y D}$, and suppose that $V$ has one of the following matrices in the basis $\{x, y\}$ (recall from the beginning of section 1.1 that $\mathbf{i}, \xi$ denote fixed roots of unity of orders 4 and 8 respectively).

$$
\begin{gathered}
b_{q}^{1}=\left(\begin{array}{rr}
-1 & q^{-1} \\
q & -1
\end{array}\right)\left(q \in \mathbf{k}^{\times}\right), \quad b^{2}=\left(\begin{array}{rr}
-1 & -1 \\
1 & -1
\end{array}\right), \\
b_{ \pm+}^{3}=\left(\begin{array}{cc}
-1 & 1 \\
\pm \mathbf{i} & -1
\end{array}\right), \quad b_{ \pm-}^{3}=\left(\begin{array}{cc}
-1 & -1 \\
\pm \mathbf{i} & -1
\end{array}\right), \\
b_{ \pm}^{4}=\left(\begin{array}{cc} 
\pm \mathbf{i} & \pm \mathbf{i} \\
-1 & -1
\end{array}\right), \quad b_{ \pm}^{5}=\left(\begin{array}{cc} 
\pm \mathbf{i} & \mp \mathbf{i} \\
-1 & -1
\end{array}\right), \\
b_{k}^{6}=\left(\begin{array}{rr}
-1 & 1 \\
\xi^{k} & -1
\end{array}\right) \quad(k=1,3,5,7) .
\end{gathered}
$$


The information about $\mathfrak{B}(V)$ that we need is given in the following table. We put $z_{1}=$ $\operatorname{Ad}_{x}(y)$ or $z_{1}=\operatorname{Ad}_{y}(x)$ depending on the case.

\begin{tabular}{|l|c|c|c|r|r|r|}
\hline \multicolumn{7}{|c|}{ TABLE 1: Matrices and algebras } \\
\hline$b$ & $\operatorname{dim} \mathfrak{B}(V)$ & $C T ?$ & $z_{1}$ & $|x|$ & $|y|$ & $\left|z_{1}\right|$ \\
\hline$b_{q}^{1}$ & 4 & Yes & 0 & 2 & 2 & \\
\hline$b^{2}$ & 8 & $Y e s$ & $\operatorname{Ad}_{x}(y)=x y+y x$ & 2 & 2 & 2 \\
\hline$b_{ \pm+}^{3}$ & 16 & No & $\operatorname{Ad}_{x}(y)=x y+y x$ & 2 & 2 & 4 \\
\hline$b_{ \pm-}^{3}$ & 16 & $N_{o}$ & $\operatorname{Ad}_{x}(y)=x y+y x$ & 2 & 2 & 4 \\
\hline$b_{ \pm}^{4}$ & 16 & $N o$ & $\operatorname{Ad}_{y}(x)=x y+y x$ & 4 & 2 & 2 \\
\hline$b_{ \pm}^{5}$ & $\geq 8 N(1) \geq 24$ & $N_{o}$ & $\operatorname{Ad}_{y}(x)=x y+y x$ & 4 & 2 & $N(1)$ \\
\hline$b_{k}^{6}$ & 32 & $N_{o}$ & $\operatorname{Ad}_{y}(x)=x y-y x$ & 2 & 2 & 8 \\
\hline
\end{tabular}

We denote by $|w|$ the nilpotency order of $w$, i.e. $w^{|w|}=0, w^{|w|-1} \neq 0$.

2. Suppose $V$ is 3-dimensional and has a matrix $b_{q_{1}, q_{2}, q_{3}}^{1}$ in the basis $\{x, y, z\}$, where

$$
b_{q_{1}, q_{2}, q_{3}}^{1}=\left(\begin{array}{ccc}
-1 & q_{1}^{-1} & q_{3}^{-1} \\
q_{1} & -1 & q_{2}^{-1} \\
q_{3} & q_{2} & -1
\end{array}\right) \quad\left(q_{1}, q_{2}, q_{3} \in \mathbf{k}^{\times}\right) \text {. }
$$

Hence $\mathfrak{B}(V)$ is an 8-dimensional $Q L S$, with relations

$$
x^{2}=y^{2}=z^{2}=0, \quad y x=q_{1} x y, z x=q_{3} x z, z y=q_{2} y z .
$$

Proof. The cases $b_{q}^{1}, b_{q_{1}, q_{2}, q_{3}}^{1}$ are particular cases of example 2.5.

The cases $b^{2}, b_{ \pm+}^{3}, b_{ \pm-}^{3}, b_{ \pm}^{5}$ and $b_{k}^{6}$ are particular cases of 2.11 .

For $b_{ \pm}^{4}$, it is straightforward to see that

$$
z y=y z, \quad z x=-\mathbf{i} x z
$$

and, using the same techniques than in 2.11, that there is a PBW basis given by $\left\{x^{a} y^{b} z^{c} \mid 0 \leq a<4,0 \leq b, c<2\right\}$.

Remark 2.15. Let $V$ be an object in $\mathcal{C}$, a braided abelian category. Suppose $V$ has a matrix

$$
b=\left(\begin{array}{ll}
b_{11} & b_{12} \\
b_{21} & b_{22}
\end{array}\right)
$$

in the basis $\{x, y\}$. Then it is clear that $V$ has the matrix

$$
[b]^{\rho}:=\left(\begin{array}{ll}
b_{22} & b_{21} \\
b_{12} & b_{11}
\end{array}\right)
$$

in the basis $\{y, x\}$. 
Consider now $\overline{\mathcal{C}}$ the category with same objects and morphisms as $\mathcal{C}$ but with the inverse braiding $\bar{c}=c^{-1}$. Consider $\bar{V}=V$ in $\overline{\mathcal{C}}$. Then $\bar{V}$ has a matrix

$$
[b]^{\tau}:=\left(\begin{array}{cc}
b_{11}^{-1} & b_{21}^{-1} \\
b_{12}^{-1} & b_{22}^{-1}
\end{array}\right)
$$

in the basis $\{x, y\}$. On the other hand, it can be shown that $\operatorname{dim} \mathfrak{B}(\bar{V})=\operatorname{dim} \mathfrak{B}(V)$. Notice, for instance, that $\left[b_{ \pm+}^{3}\right]^{\rho \tau}=b_{\mp+}^{3}$.

Let $M \in \mathrm{k}_{\mathbf{k} \Gamma}^{\mathrm{y}} \mathcal{Y} \mathcal{D}$ and $f \in \operatorname{Aut}(\Gamma)$. We write $(M)^{f}$ for the YD-module with the same underlying space as $M$ but with the structure given by

$$
h \rightarrow^{f} m=f^{-1}(h) \rightarrow m, \quad \delta^{f}(m)=(f \otimes \mathrm{id}) \delta(m) .
$$

Note that $M(g, \rho)^{f}=M\left(f(g), \rho \circ f^{-1}\right)$. In order to classify non isomorphic algebras, the following criterium will be applied:

Lemma 2.16. Let $V=\oplus_{i=1}^{n} M\left(g_{i}, \rho_{i}\right)$ and $V^{\prime}=\oplus_{i=1}^{n^{\prime}} M\left(g_{i}^{\prime}, \rho_{i}^{\prime}\right)$, where $\rho_{i}$ and $\rho_{i}^{\prime}$ are irreducible. Then $\mathfrak{B}(V) \# \mathbf{k} \Gamma \simeq \mathfrak{B}\left(V^{\prime}\right) \# \mathbf{k} \Gamma$ if and only if $n=n^{\prime}$ and there exist $f \in \operatorname{Aut}(\Gamma), \sigma \in \mathbb{S}_{n}$ such that $M\left(g_{i}^{\prime}, \rho_{i}^{\prime}\right) \simeq\left(M\left(g_{\sigma(i)}, \rho_{\sigma(i)}\right)\right)^{f}$.

Proof. Mimic [AS2, Prop. 6.3], where it is proved for $\Gamma$ abelian.

For the last step of the lifting procedure we shall use the following Proposition, which gives an invariant way to lift the elements of the bosonized algebras.

Proposition 2.17. Let $A$ be a pointed Hopf algebra, $H=\operatorname{gr} A$ be its associated graded Hopf algebra and $\Gamma$ the group of group-likes of $A$. Let $V=V(A) \in \underset{\mathbf{k} \Gamma}{\mathbf{Y} \Gamma \mathcal{D}}$ be the YD-module associated to $A$ as in (1.2.1), that is, $V=(H(1))^{\mathrm{co} H(0)}$. We have $H(1)=V \# \mathbf{k} \Gamma$. Take on $H(1)$ the $\Gamma$ action given by

$$
g \cdot(v \# h)=(g \rightarrow v) \# g h g^{-1}
$$

Consider on $A$ the adjoint action of $\Gamma$. It restricts to the coradical filtration, and then $A_{1}$ becomes a $\mathbf{k} \Gamma$-module. Let $p_{0}: A_{1} \rightarrow H(1)=A_{1} / A_{0}$ be the canonical projection. Then

1. $p_{0}$ is a morphism of $\mathbf{k} \Gamma$-modules.

2. Let $\lambda \in \mathbf{k}^{\Gamma}, \lambda(g)=\delta_{g, 1}$. Then $p=(\mathrm{id} \# \lambda) \circ p_{0}: A_{1} \rightarrow V$ is a morphism of $\mathbf{k} \Gamma$-modules.

3. There exists a section $s: V \rightarrow A_{1}$ of $\mathbf{k} \Gamma$-modules to $p$ such that if $\delta(x)=g \otimes x$ then $s(x) \in \mathcal{P}_{g, 1}(A)$.

Proof. The items 1 and 2 are straightforward looking at the definition of the action $\rightarrow$. We prove 3. For $g, h \in \Gamma$, let $\mathcal{P}_{g, h}^{\prime} \subset A_{1}$ be subspaces such that $\mathcal{P}_{g, h}(A)=\mathbf{k}(g-h) \oplus \mathcal{P}_{g, h}^{\prime}$. Then, by the Taft-Wilson theorem,

$$
A_{1}=\mathbf{k} \Gamma \oplus\left(\bigoplus_{g, h \in \Gamma} \mathcal{P}_{g, h}^{\prime}\right)
$$


We have the exact sequence of vector spaces

$$
0 \rightarrow \mathbf{k} \Gamma=A_{0} \rightarrow A_{1} \rightarrow V \# \mathbf{k} \Gamma \rightarrow 0 .
$$

Consider now the inclusion

$$
i: \oplus_{g \in \Gamma} \mathcal{P}_{g, 1}^{\prime} \hookrightarrow A_{1} .
$$

It is easy to verify that the composition $p \circ i$ is an isomorphism of vector spaces (a word of warning is needed here: $\oplus_{g \in \Gamma} \mathcal{P}_{g, 1}^{\prime}$ need not to be a $\mathbf{k} \Gamma$-submodule of $A_{1}$ ). Let $s_{0}: V \rightarrow \oplus_{g \in \Gamma} \mathcal{P}_{g, 1}^{\prime}$, $s_{0}=(p \circ i)^{-1}$, and consider as usual

$$
s: V \rightarrow A_{1}, \quad s=\frac{1}{|\Gamma|} \sum_{h \in \Gamma}\left(h \cdot s_{0}\right), \text { i.e. } s(x)=\frac{1}{|\Gamma|} \sum_{h \in \Gamma} h \cdot\left(s_{0}\left(h^{-1}-x\right)\right) .
$$

It is clear that $s$ is a $\mathbf{k} \Gamma$ section to $p$.

Let $V^{g}=\{x \in V \mid \delta(x)=g \otimes x\}$; then $V=\oplus_{g \in \Gamma} V^{g}$. Moreover, let $a \in \mathcal{P}_{g, 1}^{\prime}$. Then $\Delta a=g \otimes a+a \otimes 1$, and if $b=a+\mathrm{k} \Gamma \in A_{1} / A_{0}$ is the image of $a$ under $A_{1} \rightarrow A_{1} / A_{0}$, we have $\Delta b=g \otimes b+b \otimes 1$. Then $b \in(V \# \mathbf{k} \Gamma)^{\mathrm{co} H(0)}$, whence $b=v \# 1$ for some $v \in V$ such that $\Delta(v \# 1)=g \otimes(v \# 1)+(v \# 1) \otimes 1$. This implies that $\delta(v)=g \otimes v$, and hence $v=($ id $\# \lambda)(v \# 1) \in$ $V^{g}$. Thus $p\left(\mathcal{P}_{g, 1}^{\prime}\right) \subseteq V^{g}$ for all $g \in \Gamma$. Now, since $p \circ i: \oplus_{g \in \Gamma} \mathcal{P}_{g, 1}^{\prime} \rightarrow \oplus_{g \in \Gamma} V^{g}$ is an isomorphism, we must have that $\left.p \circ i\right|_{\mathcal{P}_{g, 1}^{\prime}}: \mathcal{P}_{g, 1}^{\prime} \rightarrow V^{g}$ is an isomorphism, whence $s_{0}\left(V^{g}\right)=\mathcal{P}_{g, 1}^{\prime}$ for all $g \in \Gamma$.

Let now $x \in V^{g}$. We claim that $s(x) \in \mathcal{P}_{g, 1}(A)$. This is true for, by the definition of $s$, we have

$$
\begin{aligned}
s(x) & =\frac{1}{|\Gamma|} \sum_{h \in \Gamma} h \cdot s_{0}\left(h^{-1}-x\right) \in \sum_{h \in \Gamma} h \cdot s_{0}\left(V^{h^{-1} g h}\right)=\sum_{h \in \Gamma} h \cdot \mathcal{P}_{h^{-1} g h, 1}^{\prime} \\
& \subseteq \sum_{h \in \Gamma} h \cdot \mathcal{P}_{h^{-1} g h, 1}(A)=\sum_{h \in \Gamma} \mathcal{P}_{g, 1}(A)=\mathcal{P}_{g, 1}(A) .
\end{aligned}
$$

We will use the above proposition as follows:

Tactic 2.18. If $V$ has a basis $\left\{x_{1}, \ldots, x_{\theta}\right\}$ such that $x_{i} \in V^{g_{i}}$, the following proposition enables us to consider $a_{1}, \ldots, a_{\theta}$ such that $a_{i} \in \mathcal{P}_{g_{i}, 1}(A)$, the projection $A_{1} \rightarrow A_{1} / A_{0}$ sends $a_{i}$ to $\left(x_{i} \# 1\right) \in V \# \mathbf{k} \Gamma$, and if $g \in \Gamma$ acts on $V$ by some matrix $T$ in the basis $\left\{x_{i}\right\}$, then $g$ acts on the linear span $\left(a_{1}, \ldots, a_{\theta}\right)$ by the same matrix (in the basis $\left.\left\{a_{i}\right\}\right)$. Furthermore, since $V$ generates $\mathfrak{B}(V)$, the $a_{i}$ 's together with $\Gamma$ generate $A$. In general, suppose $\sum_{i}\left(\alpha_{i} x_{i, 1} \cdots x_{i, n}\right)=0 \in \mathfrak{B}^{n}(V)$. Then $b=\sum_{i}\left(\alpha_{i} a_{i, 1} \cdots a_{i, n}\right) \in A$ lies in the kernel of the projection, i.e. $b \in A_{n-1}$. Usually, we shall have $b \in \mathcal{P}_{g, 1}(A)$ for some $g \in \Gamma$, from where $b$ shall lie in $A_{1}$.

The following is a very easy but useful remark:

Remark 2.19. Let $A$ be a finite dimensional Hopf algebra such that char $\mathbf{k}$ does not divide $\operatorname{dim} A$. Let $g \in A$ be a group-like and $a \in \mathcal{P}_{g, 1}(A)$ such that $g a=\zeta a g$. Let $n$ be the order of $\zeta$. Then, by the same computations as in $2.1, a^{n} \in \mathcal{P}_{g^{n}, 1}(A)$. If $g^{n}=1$ we must have $a^{n}=0$, 
for, if not, $a$ would generate a sub Hopf algebra of dimension a power of char $\mathbf{k}$ (if $\operatorname{char} \mathbf{k}>0$ ) or $\infty$ (if char $\mathbf{k}=0$ ), leading to a contradiction.

If we are lifting a QLS, we have $a_{i} \in \mathcal{P}_{g_{i}, 1}(A)$ and $g_{i} a_{j} g_{i}^{-1}=b_{i j} a_{j}$. If $i \neq j, b_{i j} b_{j i}=1$ and $g_{i} g_{j}=g_{j} g_{i}$. Then

$$
\begin{aligned}
\Delta\left(a_{i} a_{j}-b_{i j} a_{j} a_{i}\right)= & g_{i} g_{j} \otimes a_{i} a_{j}+g_{i} a_{j} \otimes a_{i}+a_{i} g_{j} \otimes a_{j}+a_{i} a_{j} \otimes 1 \\
& -b_{i j} g_{j} g_{i} \otimes a_{j} a_{i}-b_{i j} a_{j} g_{i} \otimes a_{i}-b_{i j} g_{j} a_{i} \otimes a_{j}-b_{i j} a_{j} a_{i} \otimes 1 \\
= & g_{i} g_{j} \otimes\left(a_{i} a_{j}-b_{i j} a_{j} a_{i}\right)+\left(a_{i} a_{j}-b_{i j} a_{j} a_{i}\right) \otimes 1,
\end{aligned}
$$

from where $a_{i} a_{j}-b_{i j} a_{j} a_{i} \in \mathcal{P}_{g_{i} g_{j}, 1}(A)$. As above, if char $\mathbf{k} \backslash \operatorname{dim} A$ and $g_{i} g_{j}=1$, then $a_{i} a_{j}-$ $b_{i j} a_{j} a_{i}=0$.

\section{3. $\Gamma=C_{2}$}

Let $V=\oplus_{i} M\left(h_{i}, \chi_{i}\right)$. For $\mathfrak{B}(V)$ to have dimension 16 , we must have $b_{i i}=-1 \forall i$, where $\left(b_{i j}\right)=\left(\chi_{j}\left(h_{i}\right)\right)$ is the matrix of $V$. Thus, $V=\oplus_{i} M(g, \hat{g})$, and hence $\left(b_{i j}\right)=\hat{g}(g)=-1 \forall i j$, from where $\mathfrak{B}(V)$ is a QLS with generators $x_{1}, \ldots, x_{\theta}$ and relations $x_{i}^{2}=0, x_{i} x_{j}=-x_{j} x_{i}$. Thus, $\operatorname{dim} \mathfrak{B}(V)=2^{\theta}$, and $\theta=4$. Let $a_{1}, \ldots, a_{4}$ be as in 2.18. Then $g a_{i} g=-a_{i}$ and $a_{i} \in \mathcal{P}_{g, 1}(A)$. We have by 2.19 that $a_{i}^{2} \in \mathcal{P}(A)$, and the same is true for $a_{i} a_{j}+a_{j} a_{i}$. Hence $a_{i}^{2}=a_{i} a_{j}+a_{j} a_{i}=0$ $\forall i, j$. There exist thus only one Hopf algebra of dimension 32 with coradical $\mathbf{k} C_{2}$. It is clear, using the same reasoning, that there exists a Hopf algebra with coradical $\mathbf{k} C_{2}$ and dimension $n$ if and only if $n=2^{m}$ for some $m \in \mathbb{N}$. Furthermore, if $n=2^{m}$, there exists only one such Hopf algebra. This is the content of [N, Thm. 4.2.1], but we included here a proof for completeness.

\section{4. $\Gamma$ WITH ORDER 4}

4.1. $\Gamma=C_{2} \times C_{2}$. Necessarily the rank is 2 or 3 : for rank 1 the root $b_{11}$ has order 2 , whence $\operatorname{dim}(\mathfrak{B}(V) \# \mathbf{k} \Gamma)$ would be 8 , and rank $\geq 4$ is impossible by 2.5 . The following is a list of the irreducible YD-modules giving Nichols algebras of dimension 2, with the action of Aut $(\Gamma)$ as in the lemma 2.16. In this case, Aut $(\Gamma)$ is isomorphic to $\mathbb{S}_{3}$, generated by $f_{1}$ and $f_{2}$, where, using the notation at the end of 1.1,

$$
f_{1}=\left(g_{1} \mapsto g_{1} g_{2}, g_{2} \mapsto g_{2}\right), \quad f_{2}=\left(g_{1} \mapsto g_{1}, g_{2} \mapsto g_{1} g_{2}\right)
$$


Thus, we have

\begin{tabular}{|c|l|l|c|c|}
\hline \multicolumn{5}{|c|}{ TABLE 2: modules / Aut $(\Gamma), \Gamma=C_{2} \times C_{2}$} \\
\hline$M(h, \chi)$ & $h$ & $\chi$ & $(M(h, \chi))^{f_{1}}$ & $(M(h, \chi))^{f_{2}}$ \\
\hline$Y_{2}^{1}$ & $g_{1}$ & $\hat{g}_{1}$ & $Y_{2}^{5}$ & $Y_{2}^{2}$ \\
\hline$Y_{2}^{2}$ & $g_{1}$ & $\hat{g}_{1} \hat{g}_{2}$ & $Y_{2}^{6}$ & $Y_{2}^{1}$ \\
\hline$Y_{2}^{3}$ & $g_{2}$ & $\hat{g}_{2}$ & $Y_{2}^{4}$ & $Y_{2}^{6}$ \\
\hline$Y_{2}^{4}$ & $g_{2}$ & $\hat{g}_{1} \hat{g}_{2}$ & $Y_{2}^{3}$ & $Y_{2}^{5}$ \\
\hline$Y_{2}^{5}$ & $g_{1} g_{2}$ & $\hat{g}_{1}$ & $Y_{2}^{1}$ & $Y_{2}^{4}$ \\
\hline$Y_{2}^{6}$ & $g_{1} g_{2}$ & $\hat{g}_{2}$ & $Y_{2}^{2}$ & $Y_{2}^{3}$ \\
\hline
\end{tabular}

Consider then $V=M_{1} \oplus M_{2}=M\left(h_{1}, \chi_{1}\right) \oplus M\left(h_{2}, \chi_{2}\right)$. Since Aut $(\Gamma)$ acts transitively, we may suppose that $M_{1}=Y_{2}^{1}$. For rank 2 this gives 6 possibilities, but the cases $Y_{2}^{1} \oplus Y_{2}^{4}$ and $Y_{2}^{1} \oplus Y_{2}^{6}$ are equivalent by means of $f_{1} \circ f_{2}$. Hence, the possibilities are

\begin{tabular}{|c|l|l|l|l|l|c|}
\hline \multicolumn{7}{|c|}{ TABLE 3: Rank $2, \Gamma=C_{2} \times C_{2}$} \\
\hline$V$ & $h_{1}$ & $h_{2}$ & $\chi_{1}$ & $\chi_{2}$ & $\left(b_{i j}\right)$ & $\operatorname{dim} \mathfrak{B}(V)$ \\
\hline$V_{2}^{1}$ & $g_{1}$ & $g_{1}$ & $\hat{g}_{1}$ & $\hat{g}_{1}$ & $b_{-1}^{1}$ & 4 \\
\hline$V_{2}^{2}$ & $g_{1}$ & $g_{1}$ & $\hat{g}_{1}$ & $\hat{g}_{1} \hat{g}_{2}$ & $b_{-1}^{1}$ & 4 \\
\hline$V_{2}^{3}$ & $g_{1}$ & $g_{2}$ & $\hat{g}_{1}$ & $\hat{g}_{2}$ & $b_{1}^{1}$ & 4 \\
\hline$V_{2}^{4}$ & $g_{1}$ & $g_{2}$ & $\hat{g}_{1}$ & $\hat{g}_{1} \hat{g}_{2}$ & $b^{2}$ & 8 \\
\hline$V_{2}^{5}$ & $g_{1}$ & $g_{1} g_{2}$ & $\hat{g}_{1}$ & $\hat{g}_{1}$ & $b_{-1}^{1}$ & 4 \\
\hline
\end{tabular}

For rank 3, $V=\oplus_{i=1}^{3} M_{i}=\oplus_{i=1}^{3} M\left(h_{i}, \chi_{i}\right)$. Since the module $V_{2}^{4}$ must not appear as a submodule, we have the following possibilities:

\begin{tabular}{|c|c|c|c|c|c|c|c|c|}
\hline \multicolumn{10}{|c|}{ TABLE 4: Rank $3, \Gamma=C_{2} \times C_{2}$} \\
\hline$V$ & $h_{1}$ & $h_{2}$ & $h_{3}$ & $\chi_{1}$ & $\chi_{2}$ & $\chi_{3}$ & $\left(b_{i j}\right)$ & $\operatorname{dim} \mathfrak{B}(V)$ \\
\hline$W_{2}^{1}$ & $g_{1}$ & $g_{1}$ & $g_{1}$ & $\hat{g}_{1}$ & $\hat{g}_{1}$ & $\hat{g}_{1}$ & $b_{-1,-1,-1}^{1}$ & 8 \\
\hline$W_{2}^{2}$ & $g_{1}$ & $g_{1}$ & $g_{1}$ & $\hat{g}_{1}$ & $\hat{g}_{1}$ & $\hat{g}_{1} \hat{g}_{2}$ & $b_{-1,-1,-1}^{1}$ & 8 \\
\hline$W_{2}^{3}$ & $g_{1}$ & $g_{1}$ & $g_{1} g_{2}$ & $\hat{g}_{1}$ & $\hat{g}_{1}$ & $\hat{g}_{1}$ & $b_{-1,-1,-1}^{1}$ & 8 \\
\hline$W_{2}^{4}$ & $g_{1}$ & $g_{1}$ & $g_{2}$ & $\hat{g}_{1}$ & $\hat{g}_{1}$ & $\hat{g}_{2}$ & $b_{1,-1,-1}^{1}$ & 8 \\
\hline
\end{tabular}

We compute now the liftings.

4.1.1. $V=V_{2}^{4}$. We take $a_{1}$ and $a_{2}$ as in 2.18 . Hence we have

$$
\begin{aligned}
& \Delta\left(a_{1}\right)=g_{1} \otimes a_{1}+a_{1} \otimes 1, \quad g_{1} a_{1}=-a_{1} g_{1}, \quad g_{2} a_{1}=a_{1} g_{2} ; \\
& \Delta\left(a_{2}\right)=g_{2} \otimes a_{2}+a_{2} \otimes 1, \quad g_{1} a_{2}=-a_{2} g_{1}, \quad g_{2} a_{2}=-a_{2} g_{2} .
\end{aligned}
$$

Since $a_{1}^{2} \in \mathcal{P}(A)$, we must have $a_{1}^{2}=0$ by 2.19 . For the same reason, $a_{2}^{2}=0$. Take now

$$
a_{3}=\operatorname{Ad}_{a_{1}}\left(a_{2}\right)=a_{1} a_{2}+g_{1} a_{2} \mathcal{S}\left(a_{1}\right)=a_{1} a_{2}+a_{2} a_{1},
$$


then

$$
\begin{aligned}
\Delta\left(a_{3}\right) & =g_{1} g_{2} \otimes a_{1} a_{2}+g_{1} a_{2} \otimes a_{1}+a_{1} g_{2} \otimes a_{2}+a_{1} a_{2} \otimes 1 \\
& =g_{1} g_{2} \otimes a_{1} a_{2}-a_{2} g_{1} \otimes a_{1}+a_{1} g_{2} \otimes a_{2}+a_{1} a_{2} \otimes 1 .
\end{aligned}
$$

It is straightforward to see that $a_{3}^{2} \in \mathcal{P}(A)$, and thus $a_{3}^{2}=0$. Furthermore, it is clear from the definition of $a_{3}$ that

$$
a_{1} a_{3}=a_{3} a_{1}, a_{2} a_{3}=a_{3} a_{2},
$$

and hence the unique lifting is the bosonization $\mathfrak{B}\left(V_{2}^{2}\right) \# \mathbf{k} \Gamma$.

4.1.2. $V=W_{2}^{1}$. As in 2.18 , we can take $a_{1}, a_{2}$ and $a_{3}$ such that

$$
\Delta\left(a_{i}\right)=g_{1} \otimes a_{i}+a_{i} \otimes 1, \quad g_{1} a_{i}=-a_{i} g_{1}, \quad g_{2} a_{i}=a_{i} g_{2} .
$$

We have $a_{i}^{2} \in \mathcal{P}(A)$ and $a_{i} a_{j}+a_{j} a_{i} \in \mathcal{P}(A)$, whence $a_{i}^{2}=a_{i} a_{j}+a_{j} a_{i}=0 \forall i, j$ by 2.19. Hence, there is only one lifting: the bosonization $\mathfrak{B}\left(W_{2}^{1}\right) \# \mathbf{k} \Gamma$.

4.1.3. $V=W_{2}^{2}$. This case is the same as the previous one, with the only difference that $g_{2} a_{3}=-a_{3} g_{2}$, while $g_{2} a_{i}=a_{i} g_{2}$ for $i=1,2$. The same arguments say that there is no other lifting than the bosonization $\mathfrak{B}\left(W_{2}^{2}\right) \# \mathbf{k} \Gamma$.

4.1.4. $V=W_{2}^{3}$. We have now $g_{1} a_{i}=-a_{i} g_{1}$ and $g_{2} a_{i}=a_{i} g_{2}$. The difference with the case $V=W_{2}^{1}$ is now that $\Delta\left(a_{3}\right)=g_{1} g_{2} \otimes a_{3}+a_{3} \otimes 1$. As before, $a_{i}^{2} \in \mathcal{P}(A)$ and hence $a_{i}^{2}=0$ by 2.19. Furthermore, $a_{1} a_{2}+a_{2} a_{1} \in \mathcal{P}(A)$, from where $a_{1} a_{2}=-a_{2} a_{1}$. However $a_{i} a_{3}+a_{3} a_{i} \in \mathcal{P}_{g_{2}, 1}(A)$ for $i=1,2$ and hence, there exist $\lambda_{1}, \lambda_{2} \in \mathbf{k}$ such that $a_{i} a_{3}+a_{3} a_{i}=\lambda_{i}\left(g_{2}-1\right)$. Now, let $W^{\prime}=\left(a_{1}, a_{2}\right)$ and let $T \in \mathrm{GL}_{2}(\mathbf{k}), T=\left(T_{i j}\right)$. If we replace the basis $\left\{a_{1}, a_{2}\right\}$ by $\left\{T a_{1}, T a_{2}\right\}$ (we denote also by $T$ the automorphism of $W^{\prime}$ with matrix $T$ in the basis $\left\{a_{1}, a_{2}\right\}$ ), then the constants $\left(\begin{array}{l}\lambda_{1} \\ \lambda_{2}\end{array}\right)$ are replaced by $T\left(\begin{array}{l}\lambda_{1} \\ \lambda_{2}\end{array}\right)$. Hence, there are 2 non isomorphic liftings: they can be characterized by the rank of the morphism $W^{\prime} \rightarrow \mathbf{k}\left(g_{2}-1\right), a \mapsto a a_{3}+a_{3} a$, which can be 0 or 1. Thus we have

1. The bosonization $\mathfrak{B}\left(W_{2}^{3}\right) \# \mathbf{k} \Gamma$.

2. The algebra with the above relations plus $a_{1} a_{3}+a_{3} a_{1}=g_{2}-1, a_{2} a_{3}+a_{3} a_{2}=0$.

4.1.5. $V=W_{2}^{4}$. We have here $\Delta\left(a_{i}\right)=g_{1} \otimes a_{i}+a_{i} \otimes 1$ for $i=1,2$, and $\Delta\left(a_{3}\right)=g_{2} \otimes a_{3}+a_{3} \otimes 1$, plus $g_{1} a_{i}=-a_{i} g_{1}, g_{2} a_{i}=a_{i} g_{2}$ for $i=1,2, g_{1} a_{3}=a_{3} g_{1}, g_{2} a_{3}=-a_{3} g_{2}$. As before, $a_{i}^{2}=0$ for $i=1,2,3$, and also $a_{1} a_{2}+a_{2} a_{1}=0$. Furthermore, $a_{i} a_{3}-a_{3} a_{i} \in \mathcal{P}_{g_{1} g_{2}, 1}(A)$, from where $a_{i} a_{3}-a_{3} a_{1}=\lambda_{i}\left(g_{1} g_{2}-1\right)$ for $i=1,2$. These last conditions may add some unwanted relation which would decrease the dimension of $A$. To see which $\lambda_{i}$ 's give a 32-dimensional algebra, we use the diamond lemma:

$$
\begin{aligned}
& g_{1} a_{3} a_{1}=g_{1} a_{1} a_{3}+\lambda_{1} g_{1}\left(g_{1} g_{2}-1\right)=-a_{1} a_{3} g_{1}+\lambda_{1} g_{2}-\lambda_{1} g_{1} \\
& g_{1} a_{3} a_{1}=-a_{3} a_{1} g_{1}=-a_{1} a_{3} g_{1}-\lambda_{1}\left(g_{1} g_{2}-1\right) g_{1}=-a_{1} a_{3} g_{1}-\lambda_{1} g_{2}+\lambda_{1} g_{1},
\end{aligned}
$$


from where $\lambda_{1}=0$. An analogous work with the monomial $g_{1} a_{3} a_{2}$ tells that $\lambda_{2}=0$, and there is only one lifting: the bosonization $\mathfrak{B}\left(W_{2}^{4}\right) \# \mathbf{k} \Gamma$.

4.2. $\Gamma=C_{4}$. By similar considerations as for $\Gamma=C_{2} \times C_{2}$, the rank must be 2 or 3 . Here $\operatorname{Aut}(\Gamma) \simeq C_{2}$, generated by $f(g)=g^{3}$. The list of YD-modules giving Nichols algebras of dimension a power of 2 is

\begin{tabular}{|c|l|l|c|c|}
\hline \multicolumn{5}{|c|}{ TABLE $5:$ modules / Aut $(\Gamma), \Gamma=C_{4}$} \\
\hline$M(h, \chi)$ & $h$ & $\chi$ & $(M(h, \chi))^{f}$ & $\operatorname{dim} \mathfrak{B}(M(h, \chi))$ \\
\hline$Y_{3}^{1}$ & $g$ & $\hat{g}$ & $Y_{3}^{8}$ & 4 \\
\hline$Y_{3}^{2}$ & $g$ & $\hat{g}^{2}$ & $Y_{3}^{7}$ & 2 \\
\hline$Y_{3}^{3}$ & $g$ & $\hat{g}^{3}$ & $Y_{3}^{6}$ & 4 \\
\hline$Y_{3}^{4}$ & $g^{2}$ & $\hat{g}$ & $Y_{3}^{5}$ & 2 \\
\hline$Y_{3}^{5}$ & $g^{2}$ & $\hat{g}^{3}$ & $Y_{3}^{4}$ & 2 \\
\hline$Y_{3}^{6}$ & $g^{3}$ & $\hat{g}$ & $Y_{3}^{3}$ & 4 \\
\hline$Y_{3}^{7}$ & $g^{3}$ & $\hat{g}^{2}$ & $Y_{3}^{2}$ & 2 \\
\hline$Y_{3}^{8}$ & $g^{3}$ & $\hat{g}^{3}$ & $Y_{3}^{1}$ & 4 \\
\hline
\end{tabular}

Hence, for rank 2 we have the following non isomorphic possibilities (we exclude the cases $Y_{3}^{i} \oplus Y_{3}^{j}$ with $i, j \in\{1,3,6,8\}$ by virtue of 2.5$)$.

\begin{tabular}{|c|l|l|l|l|r|r|r|r|l|c|}
\hline \multicolumn{10}{|c|}{ TABLE 6: Rank $2, \Gamma=C_{4}$} \\
\hline$V$ & $h_{1}$ & $h_{2}$ & $\chi_{1}$ & $\chi_{2}$ & $b_{11}$ & $b_{12}$ & $b_{21}$ & $b_{22}$ & $b_{i j}$ & $\operatorname{dim} \mathfrak{B}(V)$ \\
\hline$V_{3}^{1}$ & $g$ & $g$ & $\hat{g}$ & $\hat{g}^{2}$ & $\mathbf{i}$ & -1 & $\mathbf{i}$ & -1 & {$\left[b_{-}^{4}\right]^{\tau}$} & 64 \\
\hline$V_{3}^{2}$ & $g$ & $g$ & $\hat{g}^{3}$ & $\hat{g}^{2}$ & $-\mathbf{i}$ & -1 & $-\mathbf{i}$ & -1 & {$\left[b_{+}^{4}\right]^{\tau}$} & 64 \\
\hline$V_{3}^{3}$ & $g$ & $g$ & $\hat{g}^{2}$ & $\hat{g}^{2}$ & -1 & -1 & -1 & -1 & $b_{-1}^{1}$ & 4 \\
\hline$V_{3}^{4}$ & $g$ & $g^{3}$ & $\hat{g}^{2}$ & $\hat{g}^{3}$ & -1 & $-\mathbf{i}$ & -1 & $\mathbf{i}$ & {$\left[b_{-}^{5}\right]^{\rho \tau}$} & $\geq 24$ \\
\hline$V_{3}^{5}$ & $g$ & $g^{3}$ & $\hat{g}^{2}$ & $\hat{g}$ & -1 & $\mathbf{i}$ & -1 & $-\mathbf{i}$ & {$\left[b_{+}^{5}\right]^{\rho \tau}$} & $\geq 24$ \\
\hline$V_{3}^{6}$ & $g$ & $g^{3}$ & $\hat{g}^{2}$ & $\hat{g}^{2}$ & -1 & -1 & -1 & -1 & $b_{-1}^{1}$ & 4 \\
\hline$V_{3}^{7}$ & $g$ & $g^{2}$ & $\hat{g}$ & $\hat{g}$ & $\mathbf{i}$ & $\mathbf{i}$ & -1 & -1 & $b_{+}^{4}$ & 64 \\
\hline$V_{3}^{8}$ & $g$ & $g^{2}$ & $\hat{g}$ & $\hat{g}^{3}$ & $\mathbf{i}$ & $-\mathbf{i}$ & -1 & -1 & $b_{+}^{5}$ & $\geq 24$ \\
\hline$V_{3}^{9}$ & $g$ & $g^{2}$ & $\hat{g}^{3}$ & $\hat{g}$ & $-\mathbf{i}$ & $\mathbf{i}$ & -1 & -1 & $b_{-}^{5}$ & $\geq 24$ \\
\hline$V_{3}^{10}$ & $g$ & $g^{2}$ & $\hat{g}^{3}$ & $\hat{g}^{3}$ & $-\mathbf{i}$ & $-\mathbf{i}$ & -1 & -1 & $b_{-}^{4}$ & 64 \\
\hline$V_{3}^{11}$ & $g$ & $g^{2}$ & $\hat{g}^{2}$ & $\hat{g}$ & -1 & $\mathbf{i}$ & 1 & -1 & {$\left[b_{++}^{3}\right]^{\rho}$} & 16 \\
\hline$V_{3}^{12}$ & $g$ & $g^{2}$ & $\hat{g}^{2}$ & $\hat{g}^{3}$ & -1 & $-\mathbf{i}$ & 1 & -1 & {$\left[b_{-+}^{3}\right]^{\rho}$} & 16 \\
\hline$V_{3}^{13}$ & $g^{2}$ & $g^{2}$ & $\hat{g}$ & $\hat{g}$ & -1 & -1 & -1 & -1 & $b_{-1}^{1}$ & 4 \\
\hline$V_{3}^{14}$ & $g^{2}$ & $g^{2}$ & $\hat{g}$ & $\hat{g}^{3}$ & -1 & -1 & -1 & -1 & $b_{-1}^{1}$ & 4 \\
\hline
\end{tabular}

Thus, we have no rank 2 modules over $C_{4}$ giving 8-dimensional Nichols algebras. For rank 3 the possible subcases are $V_{3}^{3}, V_{3}^{6}, V_{3}^{13}$ and $V_{3}^{14}$. We have hence the following cases: 


\begin{tabular}{|c|l|l|l|l|l|l|c|c|}
\hline \multicolumn{10}{|c|}{ TABLE $7: \operatorname{Rank} 3, \Gamma=C_{4}$} \\
\hline$V$ & $h_{1}$ & $h_{2}$ & $h_{3}$ & $\chi_{1}$ & $\chi_{2}$ & $\chi_{3}$ & $b_{i j}$ & $\operatorname{dim} \mathfrak{B}(V)$ \\
\hline$W_{3}^{1}$ & $g$ & $g$ & $g$ & $\hat{g}^{2}$ & $\hat{g}^{2}$ & $\hat{g}^{2}$ & $b_{-1,-1,-1}^{1}$ & 8 \\
\hline$W_{3}^{2}$ & $g$ & $g$ & $g^{3}$ & $\hat{g}^{2}$ & $\hat{g}^{2}$ & $\hat{g}^{2}$ & $b_{-1,-1,-1}^{1}$ & 8 \\
\hline$W_{3}^{3}$ & $g^{2}$ & $g^{2}$ & $g^{2}$ & $\hat{g}$ & $\hat{g}$ & $\hat{g}$ & $b_{-1,-1,-1}^{1}$ & 8 \\
\hline$W_{3}^{4}$ & $g^{2}$ & $g^{2}$ & $g^{2}$ & $\hat{g}$ & $\hat{g}$ & $\hat{g}^{3}$ & $b_{-1,-1,-1}^{1}$ & 8 \\
\hline
\end{tabular}

We compute now the liftings:

4.2.1. $V=W_{3}^{1}$. We take $a_{i}, i=1,2,3$ as in 2.18, i.e. $\Delta\left(a_{i}\right)=g \otimes a_{i}+a_{i} \otimes 1, g a_{i}=-a_{i} g$. Hence, by $2.19, a_{i}^{2} \in \mathcal{P}_{g^{2}, 1}(A)$, and $a_{i} a_{j}+a_{j} a_{i} \in \mathcal{P}_{g^{2}, 1}(A)$, from where there exist $\lambda_{i}, 1 \leq i \leq 6$ such that

$$
\begin{aligned}
& a_{1}^{2}=\lambda_{1}\left(g^{2}-1\right), a_{2}^{2}=\lambda_{2}\left(g^{2}-1\right), a_{3}^{2}=\lambda_{3}\left(g^{2}-1\right), \\
& a_{1} a_{2}+a_{2} a_{1}=\lambda_{4}\left(g^{2}-1\right), \\
& a_{1} a_{3}+a_{3} a_{1}=\lambda_{5}\left(g^{2}-1\right), \\
& a_{2} a_{3}+a_{3} a_{2}=\lambda_{6}\left(g^{2}-1\right) .
\end{aligned}
$$

Thus, we have a quadratic form $f: W=\left(a_{1}, a_{2}, a_{3}\right) \rightarrow \mathbf{k}\left(g^{2}-1\right), f(a)=a^{2}$. Furthermore, the basis $\left\{a_{1}, a_{2}, a_{3}\right\}$ of $W$ can be replaced by any other basis of $W$, as a result of what we must consider the equivalence class of the quadratic form $f$ in order to have non isomorphic liftings. Since char $\mathbf{k} \neq 2$ and $\mathbf{k}$ is algebraically closed, $f$ is characterized by its rank, and hence there are 4 liftings (up to isomorphism):

1. $a_{i} a_{j}+a_{j} a_{i}=0$ for $i \neq j, a_{i}^{2}=0$ for $i=1,2,3$. This is the bosonization $\mathfrak{B}\left(W_{3}^{1}\right) \# \mathbf{k} \Gamma$.

2. $a_{i} a_{j}+a_{j} a_{i}=0$ for $i \neq j, a_{i}^{2}=0$ for $i=1,2, a_{3}^{2}=\left(g^{2}-1\right)$.

3. $a_{i} a_{j}+a_{j} a_{i}=0$ for $i \neq j, a_{1}^{2}=0, a_{i}^{2}=\left(g^{2}-1\right)$ for $i=2,3$.

4. $a_{i} a_{j}+a_{j} a_{i}=0$ for $i \neq j, a_{i}^{2}=\left(g^{2}-1\right)$ for $i=1,2,3$.

4.2.2. $V=W_{3}^{2}$. Similarly, we have $a_{1}, a_{2} \in \mathcal{P}_{g, 1}(A), a_{3} \in \mathcal{P}_{g^{3}, 1}(A)$ and such that $g a_{i}=-a_{i} g$ for $i=1,2,3$. Now, $a_{i} a_{3}+a_{3} a_{i} \in \mathcal{P}(A)(i=1,2)$, whence $a_{i} a_{3}+a_{3} a_{i}=0$ for $i=1$, 2 . However, there exist $\lambda_{i}, 1 \leq i \leq 4$ such that

$$
a_{i}^{2}=\lambda_{i}\left(g^{2}-1\right)(i=1,2,3), \quad a_{1} a_{2}+a_{2} a_{1}=\lambda_{4}\left(g^{2}-1\right) .
$$

Hence we have two quadratic forms:

$$
\begin{aligned}
& f_{1}: W_{1}=\left(a_{1}, a_{2}\right) \rightarrow \mathbf{k}\left(g^{2}-1\right), f_{1}(a)=a^{2}, \\
& f_{2}: W_{2}=\left(a_{3}\right) \rightarrow \mathbf{k}\left(g^{2}-1\right), f_{2}(a)=a^{2} .
\end{aligned}
$$

As before, we can replace $\left\{a_{1}, a_{2}\right\}$ by another basis of $W_{1}$, and the same for $\left\{a_{3}\right\}$. As with $W_{3}^{1}$, $f_{1}$ and $f_{2}$ are characterized by their ranks, whence there are 3 possibilities for $f_{1}$ and 2 for $f_{2}$, and finally there are 6 liftings: 
1. $a_{i} a_{j}+a_{j} a_{i}=0$ for $i \neq j, a_{1}^{2}=0, a_{2}^{2}=0, a_{3}^{2}=0$. This is the bosonization $\mathfrak{B}\left(W_{3}^{2}\right) \# \mathbf{k} \Gamma$.

2. $a_{i} a_{j}+a_{j} a_{i}=0$ for $i \neq j, a_{1}^{2}=0, a_{2}^{2}=\left(g^{2}-1\right), a_{3}^{2}=0$.

3. $a_{i} a_{j}+a_{j} a_{i}=0$ for $i \neq j, a_{1}^{2}=\left(g^{2}-1\right), a_{2}^{2}=\left(g^{2}-1\right), a_{3}^{2}=0$.

4. $a_{i} a_{j}+a_{j} a_{i}=0$ for $i \neq j, a_{1}^{2}=0, a_{2}^{2}=0, a_{3}^{2}=\left(g^{2}-1\right)$.

5. $a_{i} a_{j}+a_{j} a_{i}=0$ for $i \neq j, a_{1}^{2}=0, a_{2}^{2}=\left(g^{2}-1\right), a_{3}^{2}=\left(g^{2}-1\right)$.

6. $a_{i} a_{j}+a_{j} a_{i}=0$ for $i \neq j, a_{1}^{2}=\left(g^{2}-1\right), a_{2}^{2}=\left(g^{2}-1\right), a_{3}^{2}=\left(g^{2}-1\right)$.

4.2.3. $V=W_{3}^{3}$. The situation here is easier: we have $a_{i} \in \mathcal{P}_{g^{2}, 1}(A)$ and $g a_{i}=\mathbf{i} a_{i} g$ for $i=1,2,3$. Since $a_{i}^{2} \in \mathcal{P}(A)$ and $a_{i} a_{j}+a_{j} a_{i} \in \mathcal{P}(A)$ for all $i, j$, by 2.19 we have $a_{i}^{2}=a_{i} a_{j}+a_{j} a_{i}=0$, and there is only one lifting: the bosonization $\mathfrak{B}\left(W_{3}^{3}\right) \# \mathbf{k} \Gamma$.

4.2.4. $V=W_{3}^{4}$. Similar to the previous case, the only difference being that $g a_{i}=\mathbf{i} a_{i} g$ for $i=1,2$ and $g a_{3}=-\mathbf{i} a_{3} g$. There is only one lifting: the bosonization $\mathfrak{B}\left(W_{3}^{3}\right) \# \mathbf{k} \Gamma$.

\section{5. $\Gamma$ OF ORDER 8}

By 2.8 we have $\operatorname{dim} V \leq 2$. If $\operatorname{dim} V=1$, we have $V=M(h, \chi), h \in Z(\Gamma)$ and $\chi$ a character such that $\chi(h)$ has order 4 . If $\Gamma$ is non abelian, we have $Z(\Gamma) \subseteq[\Gamma ; \Gamma]$, whence any irreducible representation of degree 1 vanishes on the center, and $\chi(h)=1$. This tells that there are no 32-dimensional pointed Hopf algebras with non abelian coradical of dimension 8.

Remark 5.0.1. In turn this explains why there are no 16-dimensional pointed Hopf algebras with non abelian coradical (see $[\mathrm{CDR}]$ ).

If $\operatorname{dim} V=2$. Then, by [AG, Prop. 3.1.11], $V$ comes from the abelian case, and hence it has a matrix $\left(b_{i j}\right)$. By 2.5, $V$ must be a QLS over some subgroup $\Gamma^{\prime} \subseteq \Gamma$, with $b_{11}=b_{22}=-1$. If $\Gamma$ is non abelian, there are in principle three possibilities for $V$ to be 2-dimensional:

5.0.2. Decomposition of $V, \Gamma$ non abelian.

1. $V=M\left(h_{1}, \chi_{1}\right) \oplus M\left(h_{2}, \chi_{2}\right)$, where the $h_{i}$ 's are central in $\Gamma$ and the $\chi_{i}$ 's are characters,

2. $V=M(h, \chi)$, where $\left[\Gamma: \Gamma_{h}\right]=2$ and $\chi$ is a character of $\Gamma_{h}$,

3. $V=M(h, \rho)$, where $h$ is central in $\Gamma$ and $\operatorname{dim} \rho=2$.

Case 1 does not arise for the same reasons as above: any irreducible representation of degree 1 of a non abelian group of order 8 vanishes on the center, and we would have $b_{11}=b_{22}=1$, contradicting 2.2. We shall proceed as follows:

Tactic 5.0.3. In case 2 , let $x$ be a generator of the space affording $\chi$. Note that $\chi(h)=b_{11}=$ -1 . Let $t \notin \Gamma_{h}$. The conjugacy class of $h$ is $\left\{h,{ }^{t} h=t h t^{-1}\right\}$, and since $\left[\Gamma: \Gamma_{h}\right]=2$ it is easy to 
prove that ${ }^{t} h \in \Gamma_{h}$. Notice that $t^{-1} \notin \Gamma_{h}$, whence $t^{-1} h t=t h t^{-1}={ }^{t} h$. Let $y=t \rightarrow x$ and let $\zeta=\chi\left({ }^{t} h\right)$. Then $\delta(y)={ }^{t} h \otimes y$, and $c$ can be computed as follows:

$$
\begin{aligned}
c(x \otimes x) & =(h \rightarrow x) \otimes x=-x \otimes x, \\
c(x \otimes y) & =(h \rightarrow y) \otimes x=(h t \rightarrow x) \otimes x=\left(t t^{-1} h t \rightarrow x\right) \otimes x \\
& =(\zeta t \rightarrow x) \otimes x=\zeta y \otimes x, \\
c(y \otimes x) & =\left({ }^{t} h \rightarrow x\right) \otimes y=\zeta x \otimes y, \\
c(y \otimes y) & =\left({ }^{t} h t \rightarrow x\right) \otimes y=(t h \rightarrow x) \otimes y=-y \otimes y .
\end{aligned}
$$

Therefore, the matrix of $c$ is $\left(\begin{array}{rr}-1 & \zeta \\ \zeta & -1\end{array}\right)$, whence $1=b_{12} b_{21}=\zeta^{2}$, i.e. $\zeta= \pm 1$. To compute the liftings, let $a, b$ be liftings of $x, y$ as in 2.18. Then $h a h^{-1}=-a$ and $b=t a t^{-1}$. The action of $\Gamma$ on $A_{1}$ may be computed from the action of $\Gamma$ on $V$, as explained in 2.18.

Tactic 5.0.4. In case 3 , let $x$ be a generator of the space affording $\rho$, and let $t \in \Gamma$ such that $y=\rho(t)(x)$ is linearly independent with $x$. Since $\rho$ is irreducible and $h$ is central, $\rho(h)=\zeta$ id for some $\zeta \in \mathbf{k}^{\times}$. Then the matrix of $c$ is $\left(\begin{array}{cc}\zeta & \zeta \\ \zeta & \zeta\end{array}\right)$, from where $\zeta=b_{11}=-1$. Let $a, b$ be liftings of $x, y$ as in 2.18. Then $b=t a t^{-1}$ and $h b h^{-1}=-b$.

Remark 5.0.5. These cases do not arise when classifying pointed Hopf algebras of dimension $p^{5}, p$ an odd prime number (see $[\mathrm{G}]$ ), and there are no pointed Hopf algebras of dimension $p^{5}$ with non commutative coradical of dimension $p^{3}$.

We consider now the case $\Gamma$ abelian.

\subsubsection{Use of diamond lemma}

If $\Gamma$ is abelian and $\operatorname{dim} V=1$, then $V=M(h, \chi)$ and, as in 2.18, we have $a=a_{1} \in \mathcal{P}_{h, 1}(A)$. Furthermore, for $\mathfrak{B}(V)$ to be 4-dimensional, we must have $\chi(h)$ of order 4. By 2.19, $a^{4} \in$ $\mathcal{P}_{h^{4}, 1}(A)=\mathbf{k}\left(h^{4}-1\right)\left(\chi(h)\right.$ having order 4 , this implies that $h^{4}$ does not coincide neither with $h, h^{2}$ nor $h^{3}$ ). We have hence $A$ generated by $\Gamma$ and $a$, with relations

$$
t_{a t}{ }^{-1}=\chi(t) a \forall t \in \Gamma, \quad a^{4}=\lambda\left(h^{4}-1\right) .
$$

If $h^{4} \neq 1$, taking a suitable scalar multiple of $a$ we may suppose that $\lambda \in\{0,1\}$. We use the diamond lemma to prove that $A$ is 32-dimensional, but this adds some condition on $\lambda$. Let $t \in \Gamma$. We have

$$
\begin{aligned}
& t a^{4}=\chi^{4}(t) a^{4} t=\chi^{4}(t) \lambda\left(h^{4}-1\right) t, \\
& t a^{4}=t \lambda\left(h^{4}-1\right)=\lambda\left(h^{4}-1\right) t,
\end{aligned}
$$

whence

$$
\lambda\left(\chi^{4}(t)-1\right)\left(h^{4}-1\right)=0 \forall t \in \Gamma
$$


If $h^{4}=1$ we can take $\lambda=0$, and hence condition (5.0.7) can be written in the more compact form

$$
\lambda\left(\chi^{4}-1\right)=0
$$

If $\Gamma$ is abelian and $\operatorname{dim} V=2$, then $V=M\left(h_{1}, \chi_{1}\right) \oplus M\left(h_{2}, \chi_{2}\right)$. We have as in 2.18 $a_{i} \in \mathcal{P}_{h_{i}, 1}(A), t a_{i} t^{-1}=\chi_{i}(t) a_{i}$. As in 2.19, $a_{i}^{2} \in \mathcal{P}_{h_{i}^{2}, 1}(A)$ and $\operatorname{Ad}_{a_{1}}\left(a_{2}\right)=a_{1} a_{2}-\chi_{2}\left(h_{1}\right) a_{2} a_{1} \in$ $\mathcal{P}_{h_{1} h_{2}, 1}(A)$. Since $\mathfrak{B}(V)$ is 4 -dimensional, by 2.5 it is a $\mathrm{QP}$, and we have $\chi_{2}\left(h_{1}\right) \chi_{1}\left(h_{2}\right)=1$, $\chi_{i}\left(h_{i}\right)=-1$. Now, $h_{1} h_{2} \neq h_{1}$ and $h_{1} h_{2} \neq h_{2}$, for if not $h_{2}=1$ or $h_{1}=1$, contradicting $\chi_{i}\left(h_{i}\right)=-1$. Moreover, if $h_{1}^{2}=h_{2}$, we would have

$$
-1=\chi_{2}\left(h_{2}\right)=\chi_{2}\left(h_{1}^{2}\right)=\left(\chi_{2}\left(h_{1}\right)\right)^{2}=\left(\chi_{1}\left(h_{2}\right)\right)^{-2}=\left(\chi_{1}\left(h_{1}\right)\right)^{-4}=(-1)^{-4}=1,
$$

which is absurd. Thus $h_{1}^{2} \neq h_{2}$, and analogously $h_{2}^{2} \neq h_{1}$.

Hence, $\mathcal{P}_{h_{i}^{2}, 1}(A)=\mathbf{k}\left(h_{i}^{2}-1\right)$ and $\mathcal{P}_{h_{1} h_{2}, 1}(A)=\mathbf{k}\left(h_{1} h_{2}-1\right)$, and we have

$$
a_{i}^{2}=\lambda_{i}\left(h_{i}^{2}-1\right)(i=1,2), \quad a_{1} a_{2}-\chi_{2}\left(h_{1}\right) a_{2} a_{1}=\lambda_{3}\left(h_{1} h_{2}-1\right) .
$$

Similarly to (5.0.8), we get

$$
\lambda_{1}\left(\chi_{1}^{2}-1\right)=0, \quad \lambda_{2}\left(\chi_{2}^{2}-1\right)=0 .
$$

Doing the same with the monomials $t a_{2} a_{1}, t \in \Gamma$, we arrive to the condition

$$
\lambda_{3}\left(\chi_{1} \chi_{2}-1\right)=0 \text {. }
$$

We consider now group by group.

5.1. $\Gamma=C_{2} \times C_{2} \times C_{2}$. There are no 1-dimensional modules $M(h, \chi)$ in ${ }_{\mathbf{k} \Gamma}^{\mathrm{k} \Gamma} \mathcal{Y} \mathcal{D}$ s.t. $\chi(h)$ has order 4 , whence the rank must be 2 . Now, the YD-modules giving 2-dimensional Nichols algebras are those $M(h, \chi)$ such that $\chi(h)=-1$, but $\operatorname{Aut}(\Gamma) \simeq \mathrm{GL}_{3}(\mathbb{Z} / 2)$, and this group acts transitively on these modules. Hence we can suppose that $M_{1}=M\left(g_{1}, \hat{g}_{1}\right)$ (we are using the notation at the end of 1.1). The subgroup of $\operatorname{Aut}(\Gamma)$ which fixes $M_{1}$ is isomorphic to $\mathbb{S}_{3}$, and is generated by

$$
f_{1}=\left(g_{1} \mapsto g_{1}, g_{2} \mapsto g_{2} g_{3}, g_{3} \mapsto g_{3}\right), \quad f_{2}=\left(g_{1} \mapsto g_{1}, g_{2} \mapsto g_{2}, g_{3} \mapsto g_{2} g_{3}\right) .
$$

Thus we consider the action of this subgroup on the modules and choose one element in each orbit:

$$
\begin{aligned}
& Y_{4}^{1}=M\left(g_{1}, \hat{g}_{1}\right), Y_{4}^{2}=M\left(g_{1}, \hat{g}_{1} \hat{g}_{2}\right), Y_{4}^{3}=M\left(g_{1} g_{2}, \hat{g}_{1}\right), Y_{4}^{4}=M\left(g_{1} g_{2}, \hat{g}_{1} \hat{g}_{3}\right), \\
& Y_{4}^{5}=M\left(g_{1} g_{2}, \hat{g}_{2}\right), Y_{4}^{6}=M\left(g_{2}, \hat{g}_{2}\right), Y_{4}^{7}=M\left(g_{2}, \hat{g}_{1} \hat{g}_{2}\right) .
\end{aligned}
$$

In order to give non isomorphic modules after taking the sums $Y_{4}^{1} \oplus Y_{4}^{i}$, we must take into account the possibility of switching the order, as in lemma 2.16. The modules $Y_{4}^{1} \oplus Y_{4}^{5}$ and $Y_{4}^{1} \oplus Y_{4}^{7}$ give isomorphic algebras when its Nichols algebras are bosonized by means of the automorphism

$$
\left(g_{1} \mapsto g_{2}, g_{2} \mapsto g_{1} g_{2}, g_{3} \mapsto g_{3}\right)
$$


and we consider only $Y_{4}^{1} \oplus Y_{4}^{5}$. Hence, the list is

\begin{tabular}{|c|l|l|l|l|l|l|l|l|l|c|}
\hline \multicolumn{10}{|c|}{ TABLE 8: Rank $2, \Gamma=C_{2} \times C_{2} \times C_{2}$} \\
\hline$V$ & $h_{1}$ & $h_{2}$ & $\chi_{1}$ & $\chi_{2}$ & $b_{11}$ & $b_{12}$ & $b_{21}$ & $b_{22}$ & $b_{i j}$ & $\operatorname{dim} \mathfrak{B}(V)$ \\
\hline$V_{4}^{1}$ & $g_{1}$ & $g_{1}$ & $\hat{g}_{1}$ & $\hat{g}_{1}$ & -1 & -1 & -1 & -1 & $b_{-1}^{1}$ & 4 \\
\hline$V_{4}^{2}$ & $g_{1}$ & $g_{1}$ & $\hat{g}_{1}$ & $\hat{g}_{1} \hat{g}_{2}$ & -1 & -1 & -1 & -1 & $b_{-1}^{1}$ & 4 \\
\hline$V_{4}^{3}$ & $g_{1}$ & $g_{1} g_{2}$ & $\hat{g}_{1}$ & $\hat{g}_{1}$ & -1 & -1 & -1 & -1 & $b_{-1}^{1}$ & 4 \\
\hline$V_{4}^{4}$ & $g_{1}$ & $g_{1} g_{2}$ & $\hat{g}_{1}$ & $\hat{g}_{1} \hat{g}_{3}$ & -1 & -1 & -1 & -1 & $b_{-1}^{1}$ & 4 \\
\hline$V_{4}^{5}$ & $g_{1}$ & $g_{1} g_{2}$ & $\hat{g}_{1}$ & $\hat{g}_{2}$ & -1 & 1 & -1 & -1 & {$\left[b^{2}\right]^{\rho}$} & 8 \\
\hline$V_{4}^{6}$ & $g_{1}$ & $g_{2}$ & $\hat{g}_{1}$ & $\hat{g}_{2}$ & -1 & 1 & 1 & -1 & $b_{1}^{1}$ & 4 \\
\hline
\end{tabular}

We compute the liftings:

5.1.1. $V=V_{4}^{1}$. We have, as in 2.18, $a_{1}, a_{2} \in \mathcal{P}_{g_{1}, 1}(A)$, and $g_{1} a_{i}=-a_{i} g_{1}, g_{2} a_{i}=a_{i} g_{2}$, $g_{3} a_{i}=a_{i} g_{3}$ for $i=1,2$. By 2.19, we have $a_{i}^{2} \in \mathcal{P}(A)$ and also $a_{1} a_{2}+a_{2} a_{1} \in \mathcal{P}(A)$, whence $a_{i}^{2}=a_{1} a_{2}+a_{2} a_{1}=0$, and there is only one lifting: the bosonization $\mathfrak{B}\left(V_{4}^{1}\right) \# \mathbf{k} \Gamma$.

5.1.2. $V=V_{4}^{2}$. It is similar to the previous one, the only difference being that $g_{2} a_{2}=-a_{2} g_{2}$. The unique lifting is the bosonization $\mathfrak{B}\left(V_{4}^{2}\right) \# \mathbf{k} \Gamma$

5.1.3. $V=V_{4}^{3}$. Here $a_{1} \in \mathcal{P}_{g_{1}, 1}(A), a_{2} \in \mathcal{P}_{g_{1} g_{2}, 1}(A)$ and $\Gamma$ acts by $g_{1} a_{i}=-a_{i} g_{1}, g_{2} a_{i}=a_{i} g_{2}$, $g_{3} a_{i}=a_{i} g_{3}$. As before, $a_{i}^{2} \in \mathcal{P}(A)$, whence $a_{i}^{2}=0$ for $i=1$, 2. However, $a_{1} a_{2}+a_{2} a_{1} \in \mathcal{P}_{g_{2}, 1}(A)$, from where as in (5.0.9) we have $a_{1} a_{2}+a_{2} a_{1}=\lambda_{3}\left(g_{2}-1\right)$. Taking a suitable scalar multiple of $a_{1}$, we may suppose that $\lambda_{3}=0$ or $\lambda_{3}=1$. There are hence two liftings:

1. $a_{i}^{2}=0, a_{1} a_{2}+a_{2} a_{1}=0$. This is the bosonization $\mathfrak{B}\left(V_{4}^{3}\right) \# \mathbf{k} \Gamma$.

2. $a_{i}^{2}=0, a_{1} a_{2}+a_{2} a_{1}=\left(g_{2}-1\right)$.

5.1.4. $V=V_{4}^{4}$. It is similar to $V_{4}^{3}$. We have $a_{1} \in \mathcal{P}_{g_{1}, 1}(A), a_{2} \in \mathcal{P}_{g_{1} g_{2}, 1}(A)$ and $\Gamma$ acts now by $g_{1} a_{i}=-a_{i} g_{1}, g_{2} a_{i}=a_{i} g_{2}, g_{3} a_{1}=a_{1} g_{3}, g_{3} a_{2}=-a_{2} g_{3}$. As before, $a_{i}^{2}=0, a_{1} a_{2}+a_{2} a_{1}=$ $\lambda_{3}\left(g_{2}-1\right)$. Now, (5.0.11) tells that $\lambda_{3}=0$. There is only one lifting: the bosonization $\mathfrak{B}\left(V_{4}^{4}\right) \# \mathbf{k} \Gamma$.

5.1.5. $V=V_{4}^{6}$. Now $a_{1} \in \mathcal{P}_{g_{1}, 1}(A), a_{2} \in \mathcal{P}_{g_{2}, 1}(A)$ and $\Gamma$ acts by

$$
g_{1} a_{1}=-a_{1} g_{1}, \quad g_{1} a_{2}=a_{2} g_{1}, \quad g_{2} a_{1}=a_{1} g_{2}, \quad g_{2} a_{2}=-a_{2} g_{2}, \quad g_{3} a_{i}=a_{i} g_{3} .
$$

As before, $a_{i}^{2} \in \mathcal{P}(A)$, whence $a_{i}^{2}=0$. Furthermore, $a_{1} a_{2}-a_{2} a_{1} \in \mathcal{P}_{g_{1} g_{2}, 1}(A)$, from where $a_{1} a_{2}-a_{2} a_{1}=\lambda_{3}\left(g_{1} g_{2}-1\right)$. Now, (5.0.11) tells that $\lambda_{3}=0$. There is only one lifting: the bosonization $\mathfrak{B}\left(V_{4}^{6}\right) \# \mathbf{k} \Gamma$.

5.2. $\Gamma=C_{2} \times C_{4}$. We have, using the notation at the end of 1.1 , Aut $(\Gamma) \simeq \mathbb{D}_{4}$ with generators

$$
f_{1}=\left(g_{1} \mapsto g_{1} g_{2}^{2}, g_{2} \mapsto g_{1} g_{2}\right), \quad f_{2}=\left(g_{1} \mapsto g_{1} g_{2}^{2}, g_{2} \mapsto g_{2}\right)
$$


with relations $f_{1}^{4}=f_{2}^{2}=1, f_{1} f_{2}=f_{2} f_{1}^{3}$. Hence there are 3 orbits in $\Gamma$ under the action of $\operatorname{Aut}(\Gamma):\left\{g_{2}, g_{1} g_{2}, g_{2}^{3}, g_{1} g_{2}^{3}\right\},\left\{g_{1}, g_{1} g_{2}^{2}\right\}$ and $\left\{g_{2}^{2}\right\}$. There are 7 orbits of irreducible YD-modules giving Nichols algebras of dimension a power of 2 . The following is a list of one element per orbit and the subgroup of $\operatorname{Aut}(\Gamma)$ fixing the chosen element.

\begin{tabular}{|c|c|l|c|c|}
\hline \multicolumn{5}{|c|}{ TABLE $9:$ modules $/ \operatorname{Aut}(\Gamma), \Gamma=C_{2} \times C_{4}$} \\
\hline$M(h, \chi)$ & $h$ & $\chi$ & $\operatorname{dim} \mathfrak{B}(M)$ & $(\operatorname{Aut}(\Gamma))_{M}$ \\
\hline$Y_{5}^{1}$ & $g_{2}$ & $\hat{g}_{2}$ & 4 & 1 \\
\hline$Y_{5}^{2}$ & $g_{2}$ & $\hat{g}_{2}^{2}$ & 2 & $\left(f_{2}\right)$ \\
\hline$Y_{5}^{3}$ & $g_{2}$ & $\hat{g}_{2}^{3}$ & 4 & 1 \\
\hline$Y_{5}^{4}$ & $g_{2}$ & $\hat{g}_{1} \hat{g}_{2}^{2}$ & 2 & $\left(f_{2}\right)$ \\
\hline$Y_{5}^{5}$ & $g_{1}$ & $\hat{g}_{1} \hat{g}_{2}^{3}$ & 2 & $\left(f_{2} f_{1}\right)$ \\
\hline$Y_{5}^{6}$ & $g_{1}$ & $\hat{g}_{1}$ & 2 & $\left(f_{1}^{2}\right)$ \\
\hline$Y_{5}^{7}$ & $g_{2}^{2}$ & $\hat{g}_{2}$ & 2 & $\left(f_{1} f_{2}\right)$ \\
\hline
\end{tabular}

Hence there are two rank 1 Nichols algebras of dimension 4 over $C_{2} \times C_{4}$. We go now to rank 2, i.e. $V=M_{1} \oplus M_{2}$. Since $\operatorname{dim} \mathfrak{B}\left(M_{i}\right)=2$, we can suppose that $M_{1}$ is one of $Y_{5}^{2}, Y_{5}^{4}, Y_{5}^{5}, Y_{5}^{6}$ 
or $Y_{5}^{7}$. We give the list, which is the hardest to compute (we take into account 2.16).

\begin{tabular}{|c|c|c|c|c|c|c|c|c|c|c|}
\hline \multicolumn{11}{|c|}{ TABLE 10: $\operatorname{Rank} 2, \Gamma=C_{2} \times C_{4}$} \\
\hline$V$ & $\overline{h_{1}}$ & $h_{2}$ & $\chi_{1}$ & $\chi_{2}$ & $b_{11}$ & $b_{12}$ & $b_{21}$ & $b_{22}$ & $b_{i j}$ & $\operatorname{dim} \mathfrak{B}(V)$ \\
\hline$V_{5}^{1}$ & $g_{2}$ & $g_{2}$ & \begin{tabular}{|l|}
$\hat{g}_{2}^{2}$ \\
\end{tabular} & $\hat{g}_{2}^{2}$ & -1 & -1 & -1 & -1 & $b_{-1}^{1}$ & 4 \\
\hline$\overline{V_{5}^{2}}$ & $\overline{g_{2}}$ & $g_{2}$ & $\hat{g}_{2}^{2}$ & $\hat{g}_{1} \hat{g}_{2}^{2}$ & -1 & -1 & -1 & -1 & $b_{-1}^{1}$ & 4 \\
\hline$V_{5}^{3}$ & $g_{2}$ & $g_{2}$ & $\hat{g}_{1} \hat{g}_{2}^{2}$ & $\hat{g}_{1} \hat{g}_{2}^{2}$ & -1 & -1 & -1 & -1 & $b_{-1}^{1}$ & 4 \\
\hline$V_{5}^{4}$ & $g_{2}$ & $g_{1} g_{2}$ & $\hat{g}_{2}^{2}$ & $\hat{g}_{2}^{2}$ & -1 & -1 & -1 & -1 & $b_{-1}^{1}$ & 4 \\
\hline$V_{5}^{5}$ & $g_{2}$ & $g_{1} g_{2}$ & $\hat{g}_{2}^{2}$ & $\hat{g}_{1}$ & -1 & 1 & -1 & -1 & {$\left[b^{2}\right]^{\rho}$} & 8 \\
\hline$V_{5}^{6}$ & $g_{2}$ & $g_{1} g_{2}$ & $\hat{g}_{1} \hat{g}_{2}^{2}$ & $\hat{g}_{1}$ & -1 & 1 & 1 & -1 & $b_{1}^{1}$ & 4 \\
\hline$V_{5}^{7}$ & $\overline{g_{2}}$ & $g_{2}^{3}$ & $\hat{g}_{2}^{2}$ & $\hat{g}_{2}^{2}$ & -1 & -1 & -1 & -1 & $b_{-1}^{1}$ & 4 \\
\hline$V_{5}^{8}$ & $g_{2}$ & $g_{2}^{3}$ & $\hat{g}_{1} \hat{g}_{2}^{2}$ & $\hat{g}_{2}^{2}$ & -1 & -1 & -1 & -1 & $b_{-1}^{1}$ & 4 \\
\hline$V_{5}^{9}$ & $g_{2}$ & $g_{2}^{3}$ & $\hat{g}_{1} \hat{g}_{2}^{2}$ & $\hat{g}_{1} \hat{g}_{2}^{2}$ & -1 & -1 & -1 & -1 & $b_{-1}^{1}$ & 4 \\
\hline$V_{5}^{10}$ & $g_{2}$ & $g_{1}$ & $\hat{g}_{2}^{2}$ & $\hat{g}_{1}$ & -1 & 1 & 1 & -1 & $b_{1}^{1}$ & 4 \\
\hline$V_{5}^{11}$ & $g_{2}$ & $\overline{g_{1}}$ & $\hat{g}_{2}^{2}$ & $\hat{g}_{1} \hat{g}_{2}^{2}$ & -1 & -1 & 1 & -1 & $b^{2}$ & 8 \\
\hline$V_{5}^{12}$ & $g_{2}$ & $g_{1}$ & $\hat{g}_{1} \hat{g}_{2}^{2}$ & $\hat{g}_{1}$ & -1 & 1 & -1 & -1 & $\begin{array}{l}{\left[b^{2}\right]^{\rho}} \\
\end{array}$ & 8 \\
\hline$V_{5}^{13}$ & $g_{2}$ & $g_{1}$ & $\hat{g}_{1} \hat{g}_{2}^{2}$ & $\hat{g}_{1} \hat{g}_{2}^{2}$ & -1 & -1 & -1 & -1 & $b_{-1}^{1}$ & 4 \\
\hline$V_{5}^{14}$ & $g_{2}$ & $g_{1}$ & $\hat{g}_{2}^{2}$ & $\hat{g}_{1} \hat{g}_{2}^{3}$ & -1 & $-\mathrm{i}$ & 1 & -1 & {$\left[b_{-+}^{3}\right]^{\rho}$} & 16 \\
\hline$V_{5}^{15}$ & $g_{2}$ & $g_{1}$ & $\hat{g}_{2}^{2}$ & $\hat{g}_{1} \hat{g}_{2}$ & -1 & $\mathbf{i}$ & 1 & -1 & {$\left[b_{++}^{3}\right]^{\rho}$} & 16 \\
\hline$V_{5}^{16}$ & $g_{2}$ & $g_{1}$ & $\hat{g}_{1} \hat{g}_{2}^{2}$ & $\hat{g}_{1} \hat{g}_{2}^{3}$ & -1 & $-\mathbf{i}$ & -1 & -1 & {$\left[b_{--}^{3}\right]^{\rho}$} & 16 \\
\hline$V_{5}^{17}$ & $g_{2}$ & $g_{1}$ & $\hat{g}_{1} \hat{g}_{2}^{2}$ & $\hat{g}_{1} \hat{g}_{2}$ & -1 & $\mathrm{i}$ & -1 & -1 & {$\left[b_{+-}^{3}\right]^{\rho}$} & 16 \\
\hline$V_{5}^{18}$ & $g_{2}$ & $g_{2}^{2}$ & \begin{tabular}{|l|}
$\hat{g}_{2}^{2}$ \\
\end{tabular} & $\hat{g}_{1} \hat{g}_{2}$ & -1 & $\mathrm{i}$ & 1 & -1 & {$\left[b_{++}^{3}\right]^{\rho}$} & 16 \\
\hline$V_{5}^{19}$ & $g_{2}$ & $g_{2}^{2}$ & $\hat{g}_{2}^{2}$ & $\hat{g}_{1} \hat{g}_{2}^{3}$ & -1 & $-\mathbf{i}$ & 1 & -1 & {$\left[b_{-+}^{3}\right]^{\rho}$} & 16 \\
\hline$V_{5}^{20}$ & $g_{2}$ & $g_{2}^{2}$ & $\hat{g}_{1} \hat{g}_{2}^{2}$ & $\hat{g}_{2}$ & -1 & $\mathrm{i}$ & 1 & -1 & {$\left[b_{++}^{3}\right]^{\rho}$} & 16 \\
\hline$V_{5}^{21}$ & $g_{2}$ & $g_{2}^{2}$ & $\hat{g}_{1} \hat{g}_{2}^{2}$ & $\hat{g}_{2}^{3}$ & -1 & $-\mathrm{i}$ & 1 & -1 & {$\left[b_{-+}^{3}\right]^{\rho}$} & 16 \\
\hline$V_{5}^{22}$ & $g_{2}^{2}$ & $g_{2}^{2}$ & $\hat{g}_{2}$ & $\hat{g}_{2}$ & -1 & -1 & -1 & -1 & $b_{-1}^{1}$ & 4 \\
\hline$V_{5}^{23}$ & $g_{2}^{2}$ & $g_{2}^{2}$ & $\hat{g}_{2}$ & $\hat{g}_{1} \hat{g}_{2}$ & -1 & -1 & -1 & -1 & $b_{-1}^{1}$ & 4 \\
\hline$V_{5}^{24}$ & $\overline{g_{2}^{2}}$ & $g_{2}^{2}$ & $\hat{g}_{2}$ & $\hat{g}_{2}^{3}$ & -1 & -1 & -1 & -1 & $b_{-1}^{1}$ & 4 \\
\hline$V_{5}^{25}$ & $g_{2}^{2}$ & $g_{1}$ & $\hat{g}_{2}$ & $\hat{g}_{1}$ & -1 & 1 & 1 & -1 & $b_{1}^{1}$ & 4 \\
\hline$V_{5}^{26}$ & $g_{2}^{2}$ & $g_{1}$ & $\hat{g}_{2}$ & $\hat{g}_{1} \hat{g}_{2}^{3}$ & -1 & -1 & 1 & -1 & $b^{2}$ & 8 \\
\hline$V_{5}^{27}$ & $g_{2}^{2}$ & $g_{1}$ & $\hat{g}_{1} \hat{g}_{2}^{3}$ & $\begin{array}{l}\hat{g}_{1} \hat{g}_{2}^{3} \\
\end{array}$ & -1 & -1 & -1 & -1 & $b_{-1}^{1}$ & 4 \\
\hline$V_{5}^{28}$ & $g_{2}^{2}$ & $g_{1}$ & $\hat{g}_{1} \hat{g}_{2}^{3}$ & $\hat{g}_{1} \hat{g}_{2}$ & -1 & -1 & -1 & -1 & $b_{-1}^{1}$ & 4 \\
\hline$V_{5}^{29}$ & $g_{2}^{2}$ & $g_{1}$ & $\hat{g}_{1} \hat{g}_{2}$ & $\hat{g}_{1}$ & -1 & 1 & -1 & -1 & {$\left[b^{2}\right]^{\rho}$} & 8 \\
\hline$V_{5}^{30}$ & $g_{2}^{2}$ & $g_{1}$ & $\hat{g}_{2}^{3}$ & $\hat{g}_{1}$ & -1 & 1 & 1 & -1 & $b_{1}^{1}$ & 4 \\
\hline$V_{5}^{31}$ & $g_{2}^{2}$ & $g_{1} g_{2}^{2}$ & $\hat{g}_{2}$ & $\hat{g}_{1}$ & -1 & 1 & -1 & -1 & {$\left[b^{2}\right]^{\rho}$} & 8 \\
\hline$V_{5}^{32}$ & $g_{1}$ & $g_{1}$ & $\hat{g}_{1} \hat{g}_{2}^{3}$ & $\hat{g}_{1} \hat{g}_{2}^{3}$ & -1 & -1 & -1 & -1 & $b_{-1}^{1}$ & 4 \\
\hline & & & & Cont & & $n$ & t & & & \\
\hline
\end{tabular}




\begin{tabular}{|c|l|l|l|l|l|l|l|l|l|c|}
\hline$V$ & $h_{1}$ & $h_{2}$ & $\chi_{1}$ & $\chi_{2}$ & $b_{11}$ & $b_{12}$ & $b_{21}$ & $b_{22}$ & $b_{i j}$ & $\operatorname{dim} \mathfrak{B}(V)$ \\
\hline$V_{5}^{33}$ & $g_{1}$ & $g_{1}$ & $\hat{g}_{1} \hat{g}_{2}^{3}$ & $\hat{g}_{1} \hat{g}_{2}$ & -1 & -1 & -1 & -1 & $b_{-1}^{1}$ & 4 \\
\hline$V_{5}^{34}$ & $g_{1}$ & $g_{1}$ & $\hat{g}_{1} \hat{g}_{2}^{3}$ & $\hat{g}_{1}$ & -1 & -1 & -1 & -1 & $b_{-1}^{1}$ & 4 \\
\hline$V_{5}^{35}$ & $g_{1}$ & $g_{1}$ & $\hat{g}_{1}$ & $\hat{g}_{1}$ & -1 & -1 & -1 & -1 & $b_{-1}^{1}$ & 4 \\
\hline$V_{5}^{36}$ & $g_{1}$ & $g_{1}$ & $\hat{g}_{1}$ & $\hat{g}_{1} \hat{g}_{2}^{2}$ & -1 & -1 & -1 & -1 & $b_{-1}^{1}$ & 4 \\
\hline$V_{5}^{37}$ & $g_{1}$ & $g_{1} g_{2}^{2}$ & $\hat{g}_{1} \hat{g}_{2}^{3}$ & $\hat{g}_{2}$ & -1 & 1 & 1 & -1 & $b_{1}^{1}$ & 4 \\
\hline$V_{5}^{38}$ & $g_{1}$ & $g_{1} g_{2}^{2}$ & $\hat{g}_{1} \hat{g}_{2}^{3}$ & $\hat{g}_{1}$ & -1 & -1 & 1 & -1 & $b^{2}$ & 8 \\
\hline$V_{5}^{39}$ & $g_{1}$ & $g_{1} g_{2}^{2}$ & $\hat{g}_{1}$ & $\hat{g}_{1}$ & -1 & -1 & -1 & -1 & $b_{-1}^{1}$ & 4 \\
\hline$V_{5}^{40}$ & $g_{1}$ & $g_{1} g_{2}^{2}$ & $\hat{g}_{1}$ & $\hat{g}_{1} \hat{g}_{2}^{2}$ & -1 & -1 & -1 & -1 & $b_{-1}^{1}$ & 4 \\
\hline
\end{tabular}

We compute the liftings. For rank 1 we have

5.2.1. $V=Y_{5}^{1}$. As in 2.18, we have $a \in \mathcal{P}_{g_{2}, 1}(A), g_{1} a=a g_{1}, g_{2} a=\mathbf{i} a g_{2}$. By 2.19, we have $a^{4}=0$ and there is only one lifting: the bosonization $\mathfrak{B}\left(Y_{5}^{1}\right) \# \mathbf{k} \Gamma$.

5.2.2. $V=Y_{5}^{3}$. Analogous to the previous one, the only difference being that $g_{2} a=-\mathbf{i} a g_{2}$. There is only one lifting: the bosonization $\mathfrak{B}\left(Y_{5}^{3}\right) \# \mathbf{k} \Gamma$.

We go now to rank 2. As explained in 5.0.6, we have $a_{1}, a_{2}$ with $a_{i} \in \mathcal{P}_{h_{i}, 1}(A), t a_{i} t^{-1}=\chi_{i}(t) a_{i}$,

$$
a_{1}^{2}=\lambda_{1}\left(h_{1}^{2}-1\right), \quad a_{2}^{2}=\lambda_{2}\left(h_{2}^{2}-1\right), \quad a_{1} a_{2}-\chi_{2}\left(h_{1}\right) a_{2} a_{1}=\lambda_{3}\left(h_{1} h_{2}-1\right) .
$$

Before computing the liftings, we notice some facts.

Remark 5.2.3. In certain cases, some element in Aut $(\Gamma)$ interchanges $M_{1}$ and $M_{2}$. In this situation the lifting corresponding to $\left(\lambda_{1}, \lambda_{2}, \lambda_{3}\right)$ is isomorphic to that corresponding to $\left(\lambda_{2}, \lambda_{1}, \pm \lambda_{3}\right)$ (the \pm sign depending on $\chi_{2}\left(h_{1}\right)$ ).

Remark 5.2.4. In some cases $M_{1}=M_{2}, h_{1}^{2} \neq 1$ and $\chi_{1}^{2}=1$. In those cases, let $W$ be the linear span of $a_{1}, a_{2}$. We can change the basis $\left\{a_{1}, a_{2}\right\}$ by any other basis of $W$, and (similarly to the case $\left.W_{3}^{1}\right)$ we have a quadratic form $f: W \rightarrow \mathbf{k}\left(h_{1}^{2}-1\right), f(a)=a^{2}$. As in that case, $A$ is characterized by the rank of this form, whence there shall be 3 liftings.

Remark 5.2.5. In the case $V_{5}^{4}$ (and $V_{6}^{2}, V_{6}^{4}$ for $\Gamma=C_{8}$ ) there is no restriction to $\lambda_{1}, \lambda_{2}$ and $\lambda_{3}$. If we replace $\left\{a_{1}, a_{2}\right\}$ by $\left\{s a_{1}, t a_{2}\right\}\left(s, t \in \mathbf{k}^{\times}\right)$, then we get an isomorphic algebra but with $\left(\lambda_{1}, \lambda_{2}, \lambda_{3}\right)$ replaced by $\left(s^{2} \lambda_{1}, t^{2} \lambda_{2}, s t \lambda_{3}\right)$. Also, 5.2.3 applies here. If $\lambda_{1} \lambda_{2} \neq 0$, taking $s^{2}=\lambda_{2} / \lambda_{1}, t=s^{-1}$, we already get that the algebra corresponding to $\left(\lambda_{1}, \lambda_{2}, \lambda_{3}\right)$ is isomorphic to the one corresponding to $\left(\lambda_{2}, \lambda_{1}, \lambda_{3}\right)$. For $\lambda_{1} \lambda_{2}=0$, though, we get new relations. There are thus infinitely many liftings, which are parametrized by the quotient variety $\mathbf{k}^{3} / \sim$, where

$$
\begin{aligned}
& \left(\lambda_{1}, \lambda_{2}, \lambda_{3}\right) \sim\left(s^{2} \lambda_{1}, t^{2} \lambda_{2}, s t \lambda_{3}\right), \quad s, t \in \mathbf{k}^{\times} . \\
& (1,0,1) \sim(0,1,1) \\
& (1,0,0) \sim(0,1,0)
\end{aligned}
$$


Remark 5.2.6. If for some reason (for instance 2.19 or (5.0.10)) $\lambda_{1}=0$ or $\lambda_{2}=0$ then taking suitable scalar multiples of $a_{1}$ or $a_{2}$ we can suppose also that $\lambda_{3} \in\{0,1\}$.

Here is a list of the liftings up to isomorphism. We put

$$
a_{3}=a_{1} a_{2}-\chi_{2}\left(h_{1}\right) a_{2} a_{1}
$$

\begin{tabular}{|c|c|c|c|c|c|c|}
\hline \multicolumn{7}{|c|}{ TABLE 11: Rank $2, \Gamma=C_{2} \times C_{4}$, liftings } \\
\hline$V$ & Lift. & $-b_{12}$ & $a_{1}^{2}$ & $\overline{a_{2}^{2}}$ & $a_{3}$ & Remarks \\
\hline \multirow[t]{3}{*}{$V_{5}^{1}$} & \multirow[t]{3}{*}{3} & \multirow[t]{3}{*}{+} & 0 & 0 & 0 & \multirow[t]{3}{*}{ See 5.2 .4} \\
\hline & & & $g_{2}^{2}-1$ & 0 & 0 & \\
\hline & & & $g_{2}^{2}-1$ & $g_{2}^{2}-1$ & 0 & \\
\hline \multirow[t]{4}{*}{$V_{5}^{2}$} & \multirow[t]{4}{*}{4} & \multirow[t]{4}{*}{+} & 0 & 0 & 0 & \multirow[t]{4}{*}{ See 5.0.11 } \\
\hline & & & $g_{2}^{2}-1$ & 0 & 0 & \\
\hline & & & 0 & $g_{2}^{2}-1$ & 0 & \\
\hline & & & $g_{2}^{2}-1$ & $g_{2}^{2}-1$ & 0 & \\
\hline \multirow[t]{3}{*}{$V_{5}^{3}$} & \multirow[t]{3}{*}{3} & \multirow[t]{3}{*}{+} & 0 & 0 & 0 & \multirow[t]{3}{*}{ See 5.2 .4} \\
\hline & & & $g_{2}^{2}-1$ & 0 & 0 & \\
\hline & & & $g_{2}^{2}-1$ & $g_{2}^{2}-1$ & 0 & \\
\hline$V_{5}^{4}$ & $\infty$ & + & \multicolumn{3}{|c|}{$\begin{array}{c}\lambda_{1}\left(g_{2}^{2}-1\right), \lambda_{2}\left(g_{2}^{2}-1\right), \lambda_{3}\left(g_{1} g_{2}^{2}-1\right) \\
\left(\lambda_{1}, \lambda_{2}, \lambda_{3}\right) / \sim\end{array}$} & See 5.2 .5 \\
\hline \multirow[t]{3}{*}{$V_{5}^{6}$} & \multirow[t]{3}{*}{3} & \multirow[t]{3}{*}{-} & 0 & 0 & 0 & \multirow[t]{3}{*}{ See 5.0.11 and 5.2.3 } \\
\hline & & & $g_{2}^{2}-1$ & 0 & 0 & \\
\hline & & & $g_{2}^{2}-1$ & $g_{2}^{2}-1$ & 0 & \\
\hline \multirow[t]{3}{*}{$V_{5}^{7}$} & \multirow[t]{3}{*}{3} & \multirow[t]{3}{*}{+} & 0 & 0 & 0 & \multirow[t]{3}{*}{ See 2.19 and 5.2 .3} \\
\hline & & & $g_{2}^{2}-1$ & 0 & 0 & \\
\hline & & & $g_{2}^{2}-1$ & $g_{2}^{2}-1$ & 0 & \\
\hline \multirow[t]{4}{*}{$V_{5}^{8}$} & \multirow[t]{4}{*}{4} & \multirow[t]{4}{*}{+} & 0 & 0 & 0 & \multirow[t]{4}{*}{ See 2.19} \\
\hline & & & $g_{2}^{2}-1$ & 0 & 0 & \\
\hline & & & 0 & $g_{2}^{2}-1$ & 0 & \\
\hline & & & $g_{2}^{2}-1$ & $g_{2}^{2}-1$ & 0 & \\
\hline \multirow[t]{3}{*}{$V_{5}^{9}$} & \multirow[t]{3}{*}{3} & \multirow[t]{3}{*}{+} & 0 & 0 & 0 & \multirow[t]{3}{*}{ See 5.2 .3} \\
\hline & & & $g_{2}^{2}-1$ & 0 & 0 & \\
\hline & & & $g_{2}^{2}-1$ & $g_{2}^{2}-1$ & 0 & \\
\hline \multirow[t]{2}{*}{$V_{5}^{10}$} & 2 & - & 0 & 0 & 0 & See 2.19 and 5.0 .11 \\
\hline & & & $g_{2}^{2}-1$ & 0 & 0 & \\
\hline & & & & Contint & t page & \\
\hline
\end{tabular}




\begin{tabular}{|c|c|c|c|c|c|c|}
\hline$V$ & Lift. & $-b_{12}$ & $a_{1}^{2}$ & $a_{2}^{2}$ & $a_{3}$ & Remarks \\
\hline \multirow[t]{4}{*}{$V_{5}^{13}$} & \multirow[t]{4}{*}{4} & \multirow[t]{4}{*}{+} & 0 & 0 & 0 & \multirow[t]{4}{*}{ See 2.19 and 5.2 .6} \\
\hline & & & $g_{2}^{2}-1$ & 0 & 0 & \\
\hline & & & 0 & 0 & $g_{1} g_{2}-1$ & \\
\hline & & & $g_{2}^{2}-1$ & 0 & $g_{1} g_{2}-1$ & \\
\hline$V_{5}^{22}$ & 1 & + & 0 & 0 & 0 & See 2.19 \\
\hline$V_{5}^{23}$ & 1 & + & 0 & 0 & 0 & See 2.19 \\
\hline$V_{5}^{24}$ & 1 & + & 0 & 0 & 0 & See 2.19 \\
\hline$V_{5}^{25}$ & 1 & - & 0 & 0 & 0 & See 2.19 and 5.0 .11 \\
\hline$V_{5}^{27}$ & 1 & + & 0 & 0 & 0 & See 2.19 and 5.0 .11 \\
\hline \multirow[t]{2}{*}{$V_{5}^{28}$} & \multirow[t]{2}{*}{2} & \multirow[t]{2}{*}{+} & 0 & 0 & 0 & \multirow[t]{2}{*}{ See 2.19 and 5.2 .6} \\
\hline & & & 0 & 0 & $g_{1} g_{2}^{2}-1$ & \\
\hline$V_{5}^{30}$ & 1 & - & 0 & 0 & 0 & See 2.19 and 5.0 .11 \\
\hline$V_{5}^{32}$ & 1 & + & 0 & 0 & 0 & See 2.19 \\
\hline$V_{5}^{33}$ & 1 & + & 0 & 0 & 0 & See 2.19 \\
\hline$V_{5}^{34}$ & 1 & + & 0 & 0 & 0 & See 2.19 \\
\hline$V_{5}^{35}$ & 1 & + & 0 & 0 & 0 & See 2.19 \\
\hline$V_{5}^{36}$ & 1 & + & 0 & 0 & 0 & See 2.19 \\
\hline$V_{5}^{37}$ & 1 & - & 0 & 0 & 0 & See 2.19 and 5.0 .11 \\
\hline \multirow[t]{2}{*}{$V_{5}^{39}$} & \multirow[t]{2}{*}{1} & \multirow[t]{2}{*}{+} & 0 & 0 & 0 & \multirow[t]{2}{*}{ See 2.19 and 5.2 .6} \\
\hline & & & 0 & 0 & $g_{2}^{2}-1$ & \\
\hline$V_{5}^{40}$ & 1 & + & 0 & 0 & 0 & See 2.19 and 5.0 .11 \\
\hline
\end{tabular}

5.3. $\Gamma=C_{8}$. We have $\operatorname{Aut}(\Gamma) \simeq C_{2} \times C_{2}$, generated by

$$
f_{1}=\left(g \mapsto g^{3}\right), \quad f_{2}=\left(g \mapsto g^{5}\right) .
$$

The orbits of $\Gamma$ are $\left\{g, g^{3}, g^{5}, g^{7}\right\},\left\{g^{2}, g^{6}\right\}$ and $\left\{g^{4}\right\}$. There are 12 orbits of irreducible YDmodules giving Nichols algebras of dimension a power of 2 . We choose an element in each:

\begin{tabular}{|c|c|c|c|}
\hline \multicolumn{3}{|c|}{ TABLE $12:$ modules $/ \operatorname{Aut}(\Gamma), \Gamma=C_{8}$} \\
\hline$M(h, \chi)$ & $h$ & $\chi$ & $\operatorname{dim} \mathfrak{B}(M)$ \\
\hline$Y_{6}^{1}$ & $g$ & $\hat{g}$ & 8 \\
\hline$Y_{6}^{2}$ & $g$ & $\hat{g}^{3}$ & 8 \\
\hline$Y_{6}^{3}$ & $g$ & $\hat{g}^{5}$ & 8 \\
\hline$Y_{6}^{4}$ & $g$ & $\hat{g}^{7}$ & 8 \\
\hline$Y_{6}^{5}$ & $g$ & $\hat{g}^{2}$ & 4 \\
\hline$Y_{6}^{6}$ & $g$ & $\hat{g}^{6}$ & 4 \\
\hline \multicolumn{4}{|c|}{ Continues in next page } \\
\hline
\end{tabular}




\begin{tabular}{|c|l|l|c|}
\hline$M(h, \chi)$ & $h$ & $\chi$ & $\operatorname{dim} \mathfrak{B}(M)$ \\
\hline$Y_{6}^{7}$ & $g$ & $\hat{g}^{4}$ & 2 \\
\hline$Y_{6}^{8}$ & $g^{2}$ & $\hat{g}$ & 4 \\
\hline$Y_{6}^{9}$ & $g^{2}$ & $\hat{g}^{3}$ & 4 \\
\hline$Y_{6}^{10}$ & $g^{2}$ & $\hat{g}^{2}$ & 2 \\
\hline$Y_{6}^{11}$ & $g^{2}$ & $\hat{g}^{6}$ & 2 \\
\hline$Y_{6}^{12}$ & $g^{4}$ & $\hat{g}$ & 2 \\
\hline
\end{tabular}

Hence there are 4 different 4-dimensional Nichols algebras of rank 1. Now, for rank 2, we suppose $M_{1}$ is one of $Y_{6}^{7}, Y_{6}^{10}, Y_{6}^{11}$ or $Y_{6}^{12}$. We have the following possibilities (we take into account 2.16).

\begin{tabular}{|c|c|c|c|c|c|c|c|c|c|c|}
\hline \multicolumn{11}{|c|}{ TABLE 13: Rank $2, \Gamma=C_{8}$} \\
\hline$V$ & $h_{1}$ & $h_{2}$ & $\chi_{1}$ & $\chi_{2}$ & $b_{11}$ & $b_{12}$ & $b_{21}$ & $b_{22}$ & $b_{i j}$ & $\operatorname{dim} \mathfrak{B}(V)$ \\
\hline$V_{6}^{1}$ & $g$ & $g$ & $\hat{g}^{4}$ & $\hat{g}^{4}$ & -1 & -1 & -1 & -1 & $b_{-1}^{1}$ & 4 \\
\hline$V_{6}^{2}$ & $g$ & $g^{3}$ & $\hat{g}^{4}$ & $\hat{g}^{4}$ & -1 & -1 & -1 & -1 & $b_{-1}^{1}$ & 4 \\
\hline$V_{6}^{3}$ & $g$ & $g^{7}$ & $\hat{g}^{4}$ & $\hat{g}^{4}$ & -1 & -1 & -1 & -1 & $b_{-1}^{1}$ & 4 \\
\hline$V_{6}^{4}$ & $g$ & $g^{5}$ & $\hat{g}^{4}$ & \begin{tabular}{|c|}
$\hat{g}^{4}$ \\
\end{tabular} & -1 & -1 & -1 & -1 & $b_{-1}^{1}$ & 4 \\
\hline$V_{6}^{5}$ & $g$ & $g^{2}$ & $\hat{g}^{4}$ & $\hat{g}^{2}$ & -1 & i & 1 & -1 & {$\left[b_{++}^{3}\right]^{\rho}$} & 16 \\
\hline$V_{6}^{6}$ & $g$ & $g^{2}$ & $\hat{g}^{4}$ & $\hat{g}^{6}$ & -1 & $-\mathbf{i}$ & 1 & -1 & {$\left[b_{-+}^{3}\right]^{\rho}$} & 16 \\
\hline$V_{6}^{7}$ & $g$ & $g^{6}$ & $\hat{g}^{4}$ & $\hat{g}^{2}$ & -1 & $\mathrm{i}$ & 1 & -1 & {$\left[b_{++}^{3}\right]^{\rho}$} & 16 \\
\hline$V_{6}^{8}$ & $g$ & $g^{6}$ & $\hat{g}^{4}$ & $\hat{g}^{6}$ & -1 & $-\mathbf{i}$ & 1 & -1 & {$\left[b_{-+}^{3}\right]^{\rho}$} & 16 \\
\hline$V_{6}^{9}$ & $g$ & $g^{4}$ & $\hat{g}^{4}$ & $\hat{g}$ & -1 & $\xi$ & 1 & -1 & {$\left[b_{1}^{6}\right]^{\rho}$} & 32 \\
\hline$V_{6}^{10}$ & $g$ & $g^{4}$ & $\hat{g}^{4}$ & $\hat{g}^{3}$ & -1 & $\xi^{3}$ & 1 & -1 & {$\left[b_{3}^{6}\right]^{\rho}$} & 32 \\
\hline$V_{6}^{11}$ & $g$ & $g^{4}$ & $\hat{g}^{4}$ & $\hat{g}^{7}$ & -1 & $\xi^{7}$ & 1 & -1 & {$\left[b_{7}^{6}\right]^{\rho}$} & 32 \\
\hline$V_{6}^{12}$ & $g$ & $g^{4}$ & $\hat{g}^{4}$ & $\hat{g}^{5}$ & -1 & $\xi^{5}$ & 1 & -1 & {$\left[b_{5}^{6}\right]^{\rho}$} & 32 \\
\hline$V_{6}^{13}$ & $g^{2}$ & $g^{2}$ & $\hat{g}^{2}$ & $\hat{g}^{2}$ & -1 & -1 & -1 & -1 & $b_{-1}^{1}$ & 4 \\
\hline$V_{6}^{14}$ & $g^{2}$ & $g^{2}$ & $\hat{g}^{2}$ & $\hat{g}^{6}$ & -1 & -1 & -1 & -1 & $b_{-1}^{1}$ & 4 \\
\hline$V_{6}^{15}$ & $g^{2}$ & $g^{2}$ & $\hat{g}^{6}$ & $\hat{g}^{6}$ & -1 & -1 & -1 & -1 & $b_{-1}^{1}$ & 4 \\
\hline$V_{6}^{16}$ & $g^{2}$ & $g^{6}$ & $\hat{g}^{2}$ & $\hat{g}^{6}$ & -1 & -1 & -1 & -1 & $b_{-1}^{1}$ & 4 \\
\hline$V_{6}^{17}$ & $g^{2}$ & $g^{6}$ & $\hat{g}^{6}$ & \begin{tabular}{|l|}
$\hat{g}^{6}$ \\
\end{tabular} & -1 & -1 & -1 & -1 & $b_{-1}^{1}$ & 4 \\
\hline$V_{6}^{18}$ & $g^{2}$ & $g^{6}$ & $\hat{g}^{6}$ & $\hat{g}^{2}$ & -1 & -1 & -1 & -1 & $b_{-1}^{1}$ & 4 \\
\hline$V_{6}^{19}$ & $g^{4}$ & $g^{2}$ & $\hat{g}$ & $\hat{g}^{2}$ & -1 & 1 & i & -1 & $b_{++}^{3}$ & 16 \\
\hline$V_{6}^{20}$ & $g^{4}$ & $g^{2}$ & \begin{tabular}{|l|}
$g$ \\
\end{tabular} & $\hat{g}^{6}$ & -1 & 1 & $\mathrm{i}$ & -1 & $b_{++}^{3}$ & 16 \\
\hline$V_{6}^{21}$ & $g^{4}$ & $g^{6}$ & $\hat{g}$ & $\hat{g}^{6}$ & -1 & 1 & $-\mathbf{i}$ & -1 & $b_{-+}^{3}$ & 16 \\
\hline$V_{6}^{22}$ & $g^{4}$ & $g^{6}$ & $\hat{g}$ & $\hat{g}^{2}$ & -1 & 1 & $-\mathbf{i}$ & -1 & $b_{-+}^{3}$ & 16 \\
\hline & & & & & & $\overline{i x}$ & $\bar{c}$ & paq & & \\
\hline
\end{tabular}




\begin{tabular}{|c|c|l|l|l|l|l|l|l|l|c|}
\hline$V$ & $h_{1}$ & $h_{2}$ & $\chi_{1}$ & $\chi_{2}$ & $b_{11}$ & $b_{12}$ & $b_{21}$ & $b_{22}$ & $b_{i j}$ & $\operatorname{dim} \mathfrak{B}(V)$ \\
\hline$V_{6}^{23}$ & $g^{4}$ & $g^{4}$ & $\hat{g}$ & $\hat{g}$ & -1 & -1 & -1 & -1 & $b_{-1}^{1}$ & 4 \\
\hline$V_{6}^{24}$ & $g^{4}$ & $g^{4}$ & $\hat{g}$ & $\hat{g}^{3}$ & -1 & -1 & -1 & -1 & $b_{-1}^{1}$ & 4 \\
\hline$V_{6}^{25}$ & $g^{4}$ & $g^{4}$ & $\hat{g}$ & $\hat{g}^{7}$ & -1 & -1 & -1 & -1 & $b_{-1}^{1}$ & 4 \\
\hline$V_{6}^{26}$ & $g^{4}$ & $g^{4}$ & $\hat{g}$ & $\hat{g}^{5}$ & -1 & -1 & -1 & -1 & $b_{-1}^{1}$ & 4 \\
\hline
\end{tabular}

We compute now the liftings. For rank 1, we have the following algebras, where we take $a$ as in 5.0.6.

\begin{tabular}{|c|l|l|c|c|}
\hline \multicolumn{5}{|c|}{ TABLE 14: Rank $1, \Gamma=C_{8}$} \\
\hline$V$ & $h$ & $\chi$ & $a^{4}$ & Remarks \\
\hline$Y_{6}^{5}$ & $g$ & $\hat{g}^{2}$ & 0 & \multirow{2}{*}{} \\
\cline { 4 - 4 } & & & $g^{4}-1$ & \\
\hline$Y_{6}^{6}$ & $g$ & $\hat{g}^{6}$ & 0 & \\
\cline { 4 - 4 } & & & $g^{4}-1$ & \\
\hline$Y_{6}^{8}$ & $g^{2}$ & $\hat{g}$ & 0 & See $(2.19)$ \\
\hline$Y_{6}^{9}$ & $g^{2}$ & $\hat{g}^{3}$ & 0 & See $(2.19)$ \\
\hline
\end{tabular}

For rank 2, as in 5.0.6, we have $a_{1}, a_{2}$ with $a_{i} \in \mathcal{P}_{h_{i}, 1}(A), t a_{i} t^{-1}=\chi_{i}(t) a_{i}$,

$$
a_{i}^{2}=\lambda_{i}\left(h_{i}^{2}-1\right)(i=1,2), \quad a_{1} a_{2}+a_{2} a_{1}=\lambda_{3}\left(h_{1} h_{2}-1\right)
$$

(notice that $b_{12}=\chi_{2}\left(h_{1}\right)=-1$ in all the 4-dimensional cases). Equations (5.0.10), (5.0.11) and remarks 5.2.3, 5.2.4, 5.2.5 and 5.2.6 apply here also. We put

$$
a_{3}=a_{1} a_{2}+a_{2} a_{1} .
$$

\begin{tabular}{|c|c|c|c|c|c|}
\hline \multicolumn{6}{|c|}{ TABLE 15: Rank $2, \Gamma=C_{8}$, liftings } \\
\hline$V$ & Lift. & $a_{1}^{2}$ & $a_{2}^{2}$ & $a_{3}$ & Remarks \\
\hline \multirow{3}{*}{$V_{6}^{1}$} & \multirow[t]{3}{*}{3} & 0 & 0 & 0 & \multirow[t]{3}{*}{ See 5.2 .4} \\
\hline & & $g^{2}-1$ & 0 & 0 & \\
\hline & & $g^{2}-1$ & $g^{2}-1$ & 0 & \\
\hline$V_{6}^{2}$ & $\infty$ & \multicolumn{3}{|c|}{$\begin{array}{c}\lambda_{1}\left(g^{2}-1\right), \lambda_{2}\left(g^{6}-1\right), \lambda_{3}\left(g^{4}-1\right) \\
\left(\lambda_{1}, \lambda_{2}, \lambda_{3}\right) / \sim\end{array}$} & See 5.2 .5 \\
\hline \multirow[t]{3}{*}{$V_{6}^{3}$} & \multirow[t]{3}{*}{3} & 0 & 0 & 0 & \multirow[t]{3}{*}{ See 5.2 .3} \\
\hline & & $g^{2}-1$ & 0 & 0 & \\
\hline & & $g^{2}-1$ & $g^{6}-1$ & 0 & \\
\hline$V_{6}^{4}$ & $\infty$ & \multicolumn{3}{|c|}{$\begin{array}{c}\lambda_{1}\left(g^{2}-1\right), \lambda_{2}\left(g^{2}-1\right), \lambda_{3}\left(g^{6}-1\right) \\
\left(\lambda_{1}, \lambda_{2}, \lambda_{3}\right) / \sim\end{array}$} & See 5.2 .5 \\
\hline \multicolumn{6}{|c|}{ Continues in next page } \\
\hline
\end{tabular}




\begin{tabular}{|c|c|c|c|c|l|}
\hline$V$ & Lift. & $a_{1}^{2}$ & $a_{2}^{2}$ & $a_{3}$ & Remarks \\
\hline$V_{6}^{13}$ & 1 & 0 & 0 & 0 & See 5.0.10 and 5.0.11 \\
\hline$V_{6}^{14}$ & 2 & 0 & 0 & 0 & See 5.0.10 and 5.2.6 \\
\cline { 3 - 5 } & & 0 & 0 & $g^{4}-1$ & \\
\hline$V_{6}^{15}$ & 1 & 0 & 0 & 0 & See 5.0.10 and 5.0.11 \\
\hline$V_{6}^{16}$ & 1 & 0 & 0 & 0 & See 2.19 and 5.0.10 \\
\hline$V_{6}^{17}$ & 1 & 0 & 0 & 0 & See 2.19 and 5.0.10 \\
\hline$V_{6}^{18}$ & 1 & 0 & 0 & 0 & See 5.0.10 and 5.0.11 \\
\hline$V_{6}^{23}$ & 1 & 0 & 0 & 0 & See 2.19 \\
\hline$V_{6}^{24}$ & 1 & 0 & 0 & 0 & See 2.19 \\
\hline$V_{6}^{25}$ & 1 & 0 & 0 & 0 & See 2.19 \\
\hline$V_{6}^{26}$ & 1 & 0 & 0 & 0 & See 2.19 \\
\hline
\end{tabular}

5.4. $\Gamma=\mathbb{D}_{4}$. We take $\mathbb{D}_{4}$ generated by $r$ and $\sigma$ with relations $r^{4}=\sigma^{2}=1, \sigma r=r^{3} \sigma$. The conjugacy classes of $\Gamma$ are $\{1\},\left\{r^{2}\right\},\left\{\sigma, r^{2} \sigma\right\},\left\{r \sigma, r^{3} \sigma\right\}$ and $\left\{r, r^{3}\right\}$. As explained in 5.0.2, there are two possibilities for $V$. For case 5.0.2(2), $h$ must be in a conjugacy class with 2 elements, and hence we can take $h=\sigma, h=r \sigma$ or $h=r$. However, the cases $h=\sigma$ and $h=r \sigma$ give isomorphic algebras when bosonized by means of the morphism $(r \mapsto r, \sigma \mapsto r \sigma)$, and hence we take only the cases $h=\sigma$ and $h=r$. The commutator subgroups are

$$
\Gamma_{\sigma}=\left\{1, \sigma, r^{2}, r^{2} \sigma\right\} \simeq C_{2} \times C_{2}, \quad \Gamma_{r}=\left\{1, r, r^{2}, r^{3}\right\} \simeq C_{4} .
$$

Case 5.0.2(3) arises taking $h=r^{2}$ and $\rho=\rho_{0}$ the irreducible representation given by

$$
\rho_{0}(r)=\left(\begin{array}{rr}
0 & -1 \\
1 & 0
\end{array}\right), \quad \rho_{0}(\sigma)=\left(\begin{array}{ll}
0 & 1 \\
1 & 0
\end{array}\right) .
$$

The following is the list of irreducible YD-modules giving Nichols algebras of dimension 4:

\begin{tabular}{|c|c|c|c|c|}
\hline \multicolumn{5}{|c|}{ TABLE 16: $\Gamma=\mathbb{D}_{4}$} \\
\hline$M(h, \rho)$ & $h$ & $\rho$ & $b_{12}=b_{21}$ & $\operatorname{dim} \mathfrak{B}(M)$ \\
\hline$Y_{7}^{1}$ & $\sigma$ & $\sigma \mapsto-1, \quad r^{2} \mapsto 1$ & -1 & 4 \\
\hline$Y_{7}^{2}$ & $\sigma$ & $\sigma \mapsto-1, \quad r^{2} \mapsto-1$ & 1 & 4 \\
\hline$Y_{7}^{3}$ & $r$ & $r \mapsto-1$ & -1 & 4 \\
\hline$Y_{7}^{4}$ & $r^{2}$ & $\rho_{0}$ & -1 & 4 \\
\hline
\end{tabular}

We compute the liftings. Though we can state some general conditions as in (5.0.11), it turns out to be simpler to use the diamond lemma in each case. 
5.4.1. $V=Y_{7}^{1}$. As in 5.0.3, we have $a \in \mathcal{P}_{\sigma, 1}(A)$ and $b=\operatorname{rar}^{3} \in \mathcal{P}_{r^{2} \sigma, 1}(A)$. Since

$$
\begin{aligned}
& \sigma \rightarrow x=-x, \quad r \rightarrow x=y, \quad r \rightarrow y=r^{2} \rightarrow x=x, \\
& \sigma \rightarrow y=\sigma r \rightarrow x=r^{3} \sigma \rightarrow x=r\left(r^{2} \sigma\right) \rightarrow x=-r \rightarrow x=-y,
\end{aligned}
$$

we have

$$
\sigma a \sigma=-a, \quad \operatorname{rar}^{3}=b, \quad \sigma b \sigma=-b, \quad r b r^{3}=a .
$$

By 2.19, $a^{2}=b^{2}=0$, and $a b+b a \in \mathcal{P}_{r^{2}, 1}(A)$, from where (taking a suitable scalar multiple of $a$ or $b)$, we have $a b+b a=\lambda\left(r^{2}-1\right)$, with $\lambda \in\{0,1\}$. There are hence two liftings:

1. $a^{2}=b^{2}=0, a b+b a=0$. This is the bosonization $\mathfrak{B}\left(Y_{7}^{1}\right) \# \mathbf{k} \Gamma$.

2. $a^{2}=b^{2}=0, a b+b a=\left(r^{2}-1\right)$.

5.4.2. $V=Y_{7}^{2}$. It is similar to the case $Y_{7}^{1}$ : we have $a \in \mathcal{P}_{\sigma, 1}(A)$ and $b=\operatorname{rar}^{3} \in \mathcal{P}_{r^{2} \sigma, 1}(A)$ such that

$$
\sigma a \sigma=-a, \quad \operatorname{rar}^{3}=b, \quad \sigma b \sigma=b, \quad r b r^{3}=-a .
$$

As in that case, $a^{2}=b^{2}=0$ by 2.19 , and $a b-b a=\lambda\left(r^{2}-1\right)$ with $\lambda \in\{0,1\}$, but now the diamond lemma applies:

$$
0=b b a=b a b-\lambda b\left(r^{2}-1\right)=a b b-\lambda\left(r^{2}-1\right) b-\lambda b\left(r^{2}-1\right)=2 \lambda b,
$$

from where $\lambda=0$ for $A$ to be 32 -dimensional.

There is hence only one lifting: the bosonization $\mathfrak{B}\left(Y_{7}^{2}\right) \# \mathbf{k} \Gamma$.

5.4.3. $V=Y_{7}^{3}$. By 5.0.3, we have here $a \in \mathcal{P}_{r, 1}(A), b=\sigma a \sigma \in \mathcal{P}_{r^{3}, 1}(A)$, and

$$
r a r^{3}=-a, \quad r b r^{3}=-b, \quad \sigma a \sigma=b, \quad \sigma b \sigma=a .
$$

We have now $a^{2}=\lambda_{1}\left(r^{2}-1\right), b^{2}=\lambda_{2}\left(r^{2}-1\right)$. Since $a b+b a \in \mathcal{P}(A)$ then $a b+b a=0$ by 2.19. We can take $\lambda_{i} \in\{0,1\}$, from where we have four liftings. However, the automorphism $r \mapsto r^{3}, \sigma \mapsto \sigma$ gives an isomorphism between the algebra corresponding to $\left(\lambda_{1}=0, \lambda_{2}=1\right)$ and that of $\left(\lambda_{1}=1, \lambda_{2}=0\right)$, and thus there are three non isomorphic liftings:

1. $a^{2}=0, b^{2}=0, a b+b a=0$. This is the bosonization $\mathfrak{B}\left(Y_{7}^{3}\right) \# \mathbf{k} \Gamma$.

2. $a^{2}=\left(r^{2}-1\right), b^{2}=0, a b+b a=0$.

3. $a^{2}=\left(r^{2}-1\right), b^{2}=\left(r^{2}-1\right), a b+b a=0$.

5.4.4. $V=Y_{7}^{4}$. As in 5.0 .4 (with $t=r$ ), we have here $a, b \in \mathcal{P}_{r^{2}, 1}(A)$, with

$$
r a r^{3}=b, \quad r b r^{3}=-a, \quad \sigma a \sigma=b, \quad \sigma b \sigma=a .
$$

By 2.19, we have $a^{2}=b^{2}=a b+b a=0$, and thus there is only one lifting: the bosonization $\mathfrak{B}\left(Y_{7}^{4}\right) \# \mathbf{k} \Gamma$. 
5.5. $\Gamma=\mathbb{H}$. We take $\mathbb{H}=\{e,(-e), \underline{i},-\underline{i}, \underline{j},-\underline{j}, \underline{k},-\underline{k}\}$ with the standard relations $\underline{i} \underline{j}=\underline{k}$, etc. The conjugacy classes are $\{e\},\{(-e)\},\{\underline{i},-\underline{i}\},\{\underline{j},-\underline{j}\}$ and $\{\underline{k},-\underline{k}\}$. Since the last three classes give isomorphic algebras via the morphisms

$$
(\underline{i} \mapsto \underline{j} \mapsto \underline{k} \mapsto \underline{i}) \quad \text { and } \quad(\underline{i} \mapsto-\underline{i}, \underline{j} \mapsto-\underline{j}, \underline{k} \mapsto-\underline{k}),
$$

there is only one case as in 5.0.2(2), which is given by $h=\underline{i}, \chi(\underline{i})=-1$.

There is also only one case as in 5.0.2(3), which is given by $h=(-e), \rho=\rho_{0}$, where

$$
\rho_{0}(\underline{i})=\left(\begin{array}{rr}
0 & -1 \\
1 & 0
\end{array}\right), \rho_{0}(\underline{j})=\left(\begin{array}{rr}
\mathbf{i} & 0 \\
0 & -\mathbf{i}
\end{array}\right), \rho_{0}(\underline{k})=\left(\begin{array}{cc}
0 & \mathbf{i} \\
\mathbf{i} & 0
\end{array}\right) .
$$

The list of 4-dimensional Nichols algebras giving non isomorphic algebras when bosonized is given hence by

\begin{tabular}{|c|c|c|c|c|}
\hline \multicolumn{5}{|c|}{ TABLE 17: $\Gamma=\mathbb{H}$} \\
\hline$M(h, \rho)$ & $h$ & $\rho$ & $b_{12}=b_{21}$ & $\operatorname{dim} \mathfrak{B}(M)$ \\
\hline$Y_{8}^{1}$ & $\underline{i}$ & $\underline{i} \mapsto-1$ & -1 & 4 \\
\hline$Y_{8}^{2}$ & $(-e)$ & $\rho_{0}$ & -1 & 4 \\
\hline
\end{tabular}

The liftings are:

5.5.1. $V=Y_{8}^{1}$. As in 5.0 .3 we have $a \in \mathcal{P}_{\underline{i}, 1}(A)$ and $b=\underline{j} a(-\underline{j}) \in \mathcal{P}_{-\underline{i}, 1}(A)$, with the action given by

$$
\begin{aligned}
& \underline{i} a(-\underline{i})=-a, \quad \underline{j} a(-\underline{j})=b, \quad \underline{k} a(-\underline{k})=-b, \\
& \underline{i} b(-\underline{i})=-b, \quad \underline{j} b(-\underline{j})=a, \quad \underline{k} b(-\underline{k})=-a .
\end{aligned}
$$

We have $\lambda_{1}, \lambda_{2} \in\{0,1\}$ such that $a^{2}=\lambda_{1}((-e)-1), b^{2}=\lambda_{2}((-e)-1), a b+b a \in \mathcal{P}(A) \Rightarrow$ $a b+b a=0$. The diamond lemma says, however, that $\lambda_{1}=\lambda_{2}$, since

$$
\begin{aligned}
& \underline{j} a^{2}=\lambda_{1} \underline{j}((-e)-1)=\lambda_{1}((-\underline{j})-\underline{j}), \\
& \underline{j} a^{2}=b \underline{j} a=b^{2} \underline{j}=\lambda_{2}((-e)-1) \underline{j}=\lambda_{2}((-\underline{j})-\underline{j}) .
\end{aligned}
$$

Hence, there are two liftings:

1. $a^{2}=0, b^{2}=0, a b+b a=0$. This is the bosonization $\mathfrak{B}\left(Y_{8}^{1}\right) \# \mathbf{k} \Gamma$.

2. $a^{2}=((-e)-1), b^{2}=((-e)-1), a b+b a=0$.

5.5.2. $V=Y_{8}^{2}$. As in 5.0.4 we have $a, b \in \mathcal{P}_{-e, 1}(A)$ with the action given by

$$
\begin{array}{ll}
\underline{i} a(-\underline{i})=b, \quad \underline{j} a(-\underline{j})=\mathbf{i} a, \quad \underline{k} a(-\underline{k})=\mathbf{i} b, \\
\underline{i} b(-\underline{i})=-a, \quad \underline{j} b(-\underline{j})=-\mathbf{i} b, & \underline{k} b(-\underline{k})=\mathbf{i} a .
\end{array}
$$

By 2.19, we have $a^{2}=b^{2}=0$, and $a b+b a=0$, and hence there is only one lifting: the bosonization $\mathfrak{B}\left(Y_{8}^{2}\right) \# \mathbf{k} \Gamma$. 


\section{6. $\Gamma$ OF ORDER 16}

Now $\operatorname{dim} \mathfrak{B}(V)=2$, from where we must have $V=M(h, \chi), h \in Z(\Gamma)$ and $\chi$ a character such that $\chi(h)=-1$. Hence, for $\Gamma$ to have YD-modules $V$ with $\operatorname{dim} \mathfrak{B}(V)=2$, it is necessary that $Z(\Gamma) \not \subset[\Gamma ; \Gamma]$. There are 14 groups of order 16 . Among them, 3 do not verify this condition.

As explained in 2.18, we take $a \in \mathcal{P}_{h, 1}(A)$ such that $g a=\chi(g) a g$ for $g \in \Gamma$. Furthermore, there exists $\lambda \in\{0,1\}$ such that $a^{2}=\lambda\left(h^{2}-1\right)$. Analogously to (5.0.8), for $A$ to be 32dimensional we have

$$
\lambda g\left(h^{2}-1\right)=g a^{2}=\chi^{2}(g) a^{2} g=\lambda \chi^{2}(g)\left(h^{2}-1\right) g \quad \forall g \in \Gamma
$$

whence $\lambda\left(\chi^{2}(g)-1\right)\left(h^{2}-1\right)=0$. Combining this with 2.19 we get

$$
\lambda\left(\chi^{2}-1\right)=0
$$

6.1. $\Gamma=C_{2} \times C_{2} \times C_{2} \times C_{2}$. As in the case $\Gamma=C_{2} \times C_{2} \times C_{2}$, the group Aut( $\left.\Gamma\right)$ acts transitively on the YD-modules $V$ such that $\operatorname{dim} \mathfrak{B}(V)=2$, from where we get only one algebra after bosonization. We take hence $V=M\left(g_{1}, \hat{g}_{1}\right)$. By 2.19, there is only one lifting: the bosonization $\mathfrak{B}(V) \# \mathbf{k} \Gamma$.

6.2. $\Gamma=C_{2} \times C_{2} \times C_{4}$. There are 4 orbits with respect to the action of $\operatorname{Aut}(\Gamma):\{1\},\left\{g_{3}^{2}\right\}$, $\left\{g_{1}^{i} g_{2}^{j} g_{3}^{k},(i, j) \neq(0,0), k=0,2\right\},\left\{g_{1}^{i} g_{2}^{j} g_{3}^{k}, k=1,3\right\}$ (we are using the notation at the end of 1.1). The first orbit, as always, does not give a Nichols algebra of dimension 2. For the others, we have the following list of YD-modules giving non isomorphic algebras when bosonized (we indclude only the cases $\operatorname{dim} \mathfrak{B}(V)=2$ ). We include also their corresponding liftings:

\begin{tabular}{|c|c|c|c|c|c|}
\hline \multicolumn{5}{|c|}{ TABLE 18: $\Gamma=C_{2} \times C_{2} \times C_{4}$, liftings } \\
\hline$M(h, \chi)$ & Liftings & $h$ & $\chi$ & $a^{2}$ & Remarks \\
\hline$Y_{9}^{1}$ & 1 & $g_{3}^{2}$ & $\hat{g}_{3}$ & 0 & See 2.19 \\
\hline$Y_{9}^{2}$ & 1 & $g_{1}$ & $\hat{g}_{1}$ & 0 & See 2.19 \\
\hline$Y_{9}^{3}$ & 1 & $g_{1}$ & $\hat{g}_{1} \hat{g}_{3}$ & 0 & See 2.19 \\
\hline$Y_{9}^{4}$ & 2 & $g_{3}$ & $\hat{g}_{3}^{2}$ & 0 & \\
\cline { 5 - 5 } & & & & $\left(g_{3}^{2}-1\right)$ & \\
\hline$Y_{9}^{5}$ & 2 & $g_{3}$ & $\hat{g}_{1} \hat{g}_{3}^{2}$ & 0 & \\
& & & & $\left(g_{3}^{2}-1\right)$ & \\
\hline
\end{tabular}


6.3. $\Gamma=C_{4} \times C_{4}$. The orbits are $\{1\},\left\{g_{1}^{2}, g_{1}^{2} g_{2}^{2}, g_{2}^{2}\right\},\left\{g_{1}^{i} g_{2}^{j}, 2 \not i\right.$ or $\left.2 \not \nmid j\right\}$ (we are using the notation at the end of 1.1). The possibilities are

\begin{tabular}{|c|c|c|c|c|c|}
\hline \multicolumn{5}{|c|}{ TABLE 19: $\Gamma=C_{4} \times C_{4}$, liftings } \\
\hline$M(h, \chi)$ & Liftings & $h$ & $\chi$ & $a^{2}$ & Remarks \\
\hline$Y_{10}^{1}$ & 2 & $g_{1}$ & $\hat{g}_{1}^{2}$ & 0 & \\
\cline { 4 - 5 } & & & & $\left(g_{1}^{2}-1\right)$ & \\
\hline$Y_{10}^{2}$ & 1 & $g_{1}$ & $\hat{g}_{1}^{2} \hat{g}_{2}$ & 0 & See 6.0 .1 \\
\hline$Y_{10}^{3}$ & 1 & $g_{1}^{2}$ & $\hat{g}_{1}$ & 0 & See 2.19 \\
\hline
\end{tabular}

6.4. $\Gamma=C_{2} \times C_{8}$. The orbits are $\{1\},\left\{g_{2}^{4}\right\},\left\{g_{1} g_{2}^{2}, g_{1} g_{2}^{6}\right\},\left\{g_{1}, g_{1} g_{2}^{4}\right\},\left\{g_{2}^{2}, g_{2}^{6}\right\},\left\{g_{1}^{i} g_{2}^{j}, j=\right.$ $1,3,5,7\}$. The list is

\begin{tabular}{|c|c|c|c|c|c|}
\hline \multicolumn{5}{|c|}{ TABLE $20: \Gamma=C_{2} \times C_{8}$, liftings } \\
\hline$M(h, \chi)$ & Liftings & $h$ & $\chi$ & $a^{2}$ & Remarks \\
\hline$Y_{11}^{1}$ & 1 & $g_{2}^{4}$ & $\hat{g}_{2}$ & 0 & See 2.19 \\
\hline$Y_{11}^{2}$ & 2 & $g_{1} g_{2}^{2}$ & $\hat{g}_{1}$ & 0 & \\
\cline { 5 - 6 } & & & & $\left(g_{2}^{4}-1\right)$ & \\
\hline$Y_{11}^{3}$ & 1 & $g_{1} g_{2}^{2}$ & $\hat{g}_{2}^{2}$ & 0 & See 6.0 .1 \\
\hline$Y_{11}^{4}$ & 1 & $g_{1}$ & $\hat{g}_{1}$ & 0 & See 2.19 \\
\hline$Y_{11}^{5}$ & 1 & $g_{1}$ & $\hat{g}_{1} \hat{g}_{2}^{2}$ & 0 & See 2.19 \\
\hline$Y_{11}^{6}$ & 1 & $g_{1}$ & $\hat{g}_{1} \hat{g}_{2}$ & 0 & See 2.19 \\
\hline$Y_{11}^{7}$ & 1 & $g_{2}^{2}$ & $\hat{g}_{2}^{2}$ & 0 & See 6.0 .1 \\
\hline$Y_{11}^{8}$ & 1 & $g_{2}^{2}$ & $\hat{g}_{2}^{6}$ & 0 & See 6.0 .1 \\
\hline$Y_{11}^{9}$ & 1 & $g_{2}^{2}$ & $\hat{g}_{1} \hat{g}_{2}^{2}$ & 0 & See 6.0 .1 \\
\hline$Y_{11}^{10}$ & 2 & $g_{2}$ & $\hat{g}_{2}^{4}$ & 0 & \\
\cline { 5 - 5 } & & & & $\left(g_{2}^{2}-1\right)$ & \\
\hline$Y_{11}^{11}$ & 2 & $g_{2}$ & $\hat{g}_{1} \hat{g}_{2}^{4}$ & 0 & \\
\cline { 5 - 5 } & & & & $\left(g_{2}^{2}-1\right)$ & \\
\hline & & & \multicolumn{2}{|c}{} \\
\hline
\end{tabular}

6.5. $\Gamma=C_{16}$. The orbits are

$\{1\},\left\{g^{8}\right\},\left\{g^{4}, g^{12}\right\},\left\{g^{2}, g^{6}, g^{10}, g^{14}\right\},\left\{g^{i}, \quad 2 \not i\right\}$. 
The list is

\begin{tabular}{|c|c|c|c|c|c|}
\hline \multicolumn{5}{|c|}{ TABLE 21: $\Gamma=C_{16}$, liftings } \\
\hline$M(h, \chi)$ & Liftings & $h$ & $\chi$ & $a^{2}$ & Remarks \\
\hline$Y_{12}^{1}$ & 1 & $g^{8}$ & $\hat{g}$ & 0 & See 2.19 \\
\hline$Y_{12}^{2}$ & 1 & $g^{4}$ & $\hat{g}^{2}$ & 0 & See 6.0 .1 \\
\hline$Y_{12}^{3}$ & 1 & $g^{4}$ & $\hat{g}^{6}$ & 0 & See 6.0 .1 \\
\hline$Y_{12}^{4}$ & 1 & $g^{2}$ & $\hat{g}^{4}$ & 0 & See 6.0 .1 \\
\hline$Y_{12}^{5}$ & 1 & $g^{2}$ & $\hat{g}^{12}$ & 0 & See 6.0 .1 \\
\hline$Y_{12}^{6}$ & 2 & $g$ & $\hat{g}^{8}$ & 0 & \\
\cline { 5 - 5 } & & & & $\left(g^{2}-1\right)$ & \\
\hline
\end{tabular}

6.6. Non abelian groups. As said above, the non abelian groups which verify $Z(\Gamma) \not \subset[\Gamma ; \Gamma]$ are 6 , and can be presented in the following list:

\begin{tabular}{|c|c|c|c|c|}
\hline \multicolumn{5}{|c|}{ TABLE 22: Non abelian groups of order 16 } \\
\hline Group & Generators, relations & $Z(\Gamma)$ & {$[\Gamma ; \Gamma]$} & $\Gamma_{a b}$ \\
\hline$B_{1}$ & $\left(g_{1}, g_{2}\right) /\left(g_{1}^{8}, g_{2}^{2}, g_{2} g_{1} g_{2} g_{1}^{3}\right)$ & $\left(g_{1}^{2}\right)$ & $\left(g_{1}^{4}\right)$ & $C_{4} \times C_{2}$ \\
\hline$B_{2}$ & $\left(g_{1}, g_{2}, g_{3}\right) / R_{2}$ & $\left(g_{1}\right)$ & $\left(g_{1}^{2}\right)$ & $C_{2} \times C_{2} \times C_{2}$ \\
\hline$B_{3}$ & $\left(g_{1}, g_{2}\right) /\left(g_{1}^{4}, g_{2}^{4}, g_{2} g_{1} g_{2}^{3} g_{1}\right)$ & $\left(g_{1}^{2}, g_{2}^{2}\right)$ & $\left(g_{1}^{2}\right)$ & $C_{2} \times C_{4}$ \\
\hline$B_{4}=\mathbb{D}_{4} \times C_{2}$ & $(r, \sigma, t)$ & $\left(t, r^{2}\right)$ & $\left(r^{2}\right)$ & $C_{2} \times C_{2} \times C_{2}$ \\
\hline$B_{5}$ & $\left(g_{1}, g_{2}, g_{3}\right) / R_{5}$ & $\left(g_{1}^{2}, g_{2}\right)$ & $\left(g_{2}\right)$ & $C_{4} \times C_{2}$ \\
\hline$B_{6}=\mathbb{H} \times C_{2}$ & $(\underline{i}, \underline{j}, t)$ & $(t,-e)$ & $(-e)$ & $C_{2} \times C_{2} \times C_{2}$ \\
\hline
\end{tabular}

where we put for $B_{2}$ and $B_{5}$ the subgroups $R_{2}$ and $R_{5}$ respectively, generated by the elements

$$
\begin{array}{ll}
R_{2}: & g_{1}^{4}, g_{2}^{2}, g_{3}^{2},\left[g_{1} ; g_{2}\right],\left[g_{1} ; g_{3}\right],\left[g_{3} ; g_{2}\right] g_{1}^{2}, \\
R_{5}: & g_{1}^{4}, g_{2}^{2}, g_{3}^{2},\left[g_{1} ; g_{2}\right],\left[g_{3} ; g_{2}\right],\left[g_{1} ; g_{3}\right] g_{2} .
\end{array}
$$

The name $B_{i}$ corresponds to the classification in [Bu, Sect. 118]. In these groups, we have $[\Gamma ; \Gamma] \subset Z(\Gamma),(Z(\Gamma)-[\Gamma ; \Gamma])=\left\{h_{1}, h_{2}\right\}$. Hence, there are only two possibilities for $h$ in each group.

For $\Gamma=B_{4}$, for instance, $h_{1}=t$ and $h_{2}=r^{2} t$. Since the automorphism

$$
\left(r \mapsto r, \sigma \mapsto \sigma, t \mapsto r^{2} t\right)
$$

carries $h_{1}$ to $h_{2}$, we consider only $h=t$. There are 4 characters $\chi \in \hat{\Gamma}$ such that $\chi(t)=-1$. They are given by

$$
\chi(t)=-1, \quad \chi(r)= \pm 1, \quad \chi(\sigma)= \pm 1 .
$$

However, the automorphisms

$$
\left(t \mapsto t, r \mapsto t^{i} r, \sigma \mapsto t^{j} \sigma\right) \quad i, j=0,1
$$

act transitively on these representations, whence we have only one algebra. 
Doing the same analysis with the other groups, we arrive to the following list (we take $a$ as explained at the beginning of the section):

\begin{tabular}{|c|c|c|c|c|c|c|c|}
\hline \multicolumn{8}{|c|}{ TABLE 23: $\Gamma$ non abelian of order 16 , liftings } \\
\hline Group & $M(h, \chi)$ & Lift. & $h$ & \multicolumn{2}{|c|}{$\chi$} & $a^{2}$ & Remarks \\
\hline$B_{1}$ & $Y_{13}^{1}$ & 1 & $g_{1}^{2}$ & $\chi\left(g_{1}\right)=\mathbf{i}$ & $\chi\left(g_{2}\right)=1$ & 0 & See 6.0.1 \\
\hline$B_{1}$ & $Y_{13}^{2}$ & 1 & $g_{1}^{2}$ & $\chi\left(g_{1}\right)=\mathbf{i}$ & $\chi\left(g_{2}\right)=-1$ & 0 & See 6.0 .1 \\
\hline$B_{1}$ & $Y_{13}^{3}$ & 1 & $g_{1}^{2}$ & $\chi\left(g_{1}\right)=-\mathbf{i}$ & $\chi\left(g_{2}\right)=1$ & 0 & See 6.0.1 \\
\hline$B_{1}$ & $Y_{13}^{4}$ & 1 & $g_{1}^{2}$ & $\chi\left(g_{1}\right)=-\mathbf{i}$ & $\chi\left(g_{2}\right)=-1$ & 0 & See 6.0 .1 \\
\hline \multirow[t]{2}{*}{$B_{2}$} & \multirow[t]{2}{*}{$Y_{13}^{5}$} & \multirow[t]{2}{*}{2} & \multirow[t]{2}{*}{$g_{1}$} & \multirow{2}{*}{\multicolumn{2}{|c|}{$\begin{array}{ll}\chi\left(g_{1}\right)=-1 & \chi\left(g_{2}\right)=1 \\
& \chi\left(g_{3}\right)=1\end{array}$}} & 0 & \\
\hline & & & & & & $g_{1}^{2}-1$ & \\
\hline \multirow[t]{2}{*}{$B_{2}$} & \multirow[t]{2}{*}{$\bar{Y} Y_{13}^{6}$} & \multirow[t]{2}{*}{2} & \multirow[t]{2}{*}{$g_{1}$} & \multirow{2}{*}{\multicolumn{2}{|c|}{$\begin{array}{ll}\chi\left(g_{1}\right)=-1 & \chi\left(g_{2}\right)=-1 \\
& \chi\left(g_{3}\right)=-1\end{array}$}} & 0 & \\
\hline & & & & & & $g_{1}^{2}-1$ & \\
\hline$B_{3}$ & $Y_{13}^{7}$ & 1 & $g_{2}^{2}$ & $\chi\left(g_{1}\right)=1$ & $\chi\left(g_{2}\right)=\mathbf{i}$ & 0 & See 2.19 \\
\hline$B_{3}$ & $Y_{13}^{8}$ & 1 & $g_{1}^{2} g_{2}^{2}$ & $\chi\left(g_{1}\right)=1$ & $\chi\left(g_{2}\right)=\mathbf{i}$ & 0 & See 2.19 \\
\hline$B_{4}$ & $Y_{13}^{9}$ & 1 & $t$ & $\chi(t)=-1$ & $\begin{array}{l}\chi(r)=1 \\
\chi(\sigma)=1\end{array}$ & 0 & See 2.19 \\
\hline$B_{5}$ & $Y_{13}^{10}$ & 1 & $g_{1}^{2}$ & $\chi\left(g_{1}\right)=\mathbf{i}$ & $\begin{array}{l}\chi\left(g_{2}\right)=1 \\
\chi\left(g_{3}\right)=1\end{array}$ & 0 & See 2.19 \\
\hline$B_{6}$ & $Y_{13}^{11}$ & 1 & $t$ & $\chi(t)=-1$ & $\begin{array}{l}\chi(\underline{i})=1 \\
\chi(\underline{i})=1\end{array}$ & 0 & See 2.19 \\
\hline
\end{tabular}

\section{The ClASSIFICATION IS COMPLETE}

We use the techniques of [AS2, Sect. 8]. If $A$ is a 32-dimensional pointed Hopf algebra, we apply the procedures described in 1.2 and get $R$ a braided Hopf algebra in ${ }_{\mathrm{k} \Gamma}^{\mathrm{Y}} \mathcal{Y} \mathcal{D}$ satisfying (1.1.2), (1.1.3) and (1.1.4). We may consider the (braided) dual $R^{*}$ as in [AG, Def. 2.2.2], which is a braided Hopf algebra in ${ }_{\mathbf{k} \Gamma}^{\mathbf{k} \Gamma} \mathcal{Y} \mathcal{D}$ satisfying (1.1.2), (1.1.3) and (1.1.5). Let $W=R^{*}(1)$. We get in this way the sequence of braided Hopf algebras

$$
T W \rightarrow R^{*} \rightarrow \mathfrak{B}(W),
$$

whence $\mathfrak{B}(W) \# \mathbf{k} \Gamma$ is an algebra of dimension $2^{n}$, with $n \leq 5$. If $n=5$ then $R^{*}=\mathfrak{B}(W)$, from where $R^{*}$ satisfies (1.1.4) and hence $R$ satisfies (1.1.5), whence $R$ is a Nichols algebra and $A$ is in the list above. We must prove thence that the above surjection $R^{*} \rightarrow \mathfrak{B}(W)$ is in fact a bijection. This is the same as to prove that all the relations in $\mathfrak{B}(W)$ are relations in $R^{*}$, i.e., if $z \in T W$ vanishes in $\mathfrak{B}(W)$, then it vanishes in $R^{*}$. For this, notice first that for $\Gamma$ non abelian there are no bosonizations of Nichols algebras over $\Gamma$ with dimension $2^{n}, n<5$ (see 5.0.1).

Consider then $\Gamma$ abelian. If $W$ is 1-dimensional, let $x$ be a generator of $W$. We have $c(x \otimes x)=q x \otimes x$ for some $q$ a root of unity. Let $r$ be the order of $q(q \neq 1$ because of 2.2). 
Thus, $x^{r} \in \mathcal{P}\left(R^{*}\right)$ and $c\left(x^{r} \otimes x^{r}\right)=\left(x^{r} \otimes x^{r}\right)$, whence by 2.2 we have $x^{r}=0 \in R^{*}$. This implies that $R^{*}$ verifies (1.1.4), and we are done.

Consider now $\operatorname{dim} W \geq 2$. By $2.5, \operatorname{dim} \mathfrak{B}(W) \geq 2^{\operatorname{dim} W}$, and then

$$
\operatorname{dim} \mathfrak{B}(W) \# \mathbf{k} \Gamma \geq 2^{\operatorname{dim} W}|\Gamma| \geq 4|\Gamma| .
$$

Thus, if $R$ were not a Nichols algebra, $|\Gamma|$ would be $\leq 4$.

Let $\Gamma=C_{2}$. We have $W=\oplus_{i=1}^{\theta} M(g, \hat{g})$. Then for $y \in W$ we have $y^{2} \in \mathcal{P}\left(R^{*}\right)$ and $c\left(y^{2} \otimes y^{2}\right)=y^{2} \otimes y^{2}$, from where by 2.2 we have $y^{2}=0 \forall y \in W$. These are the relations for $\mathfrak{B}(W)$, and we are done.

If $|\Gamma|=4$, the bijectivity of the projection above is a consequence of the following

Proposition 7.1. Let $\Gamma$ be a finite group, $S=\oplus_{i} S(i) \in{ }_{\mathbf{k} \Gamma}^{\mathrm{k}} \mathcal{Y} \mathcal{D}$ a graded braided Hopf algebra that verifies (1.1.3) and (1.1.5), and such that $\operatorname{dim} S=8$. Then $S$ is a Nichols algebra.

Proof. Let $V=S(1)$ and $R^{\prime}=\mathfrak{B}(V)$. We have $S \rightarrow R^{\prime}$ the canonical projection. If $\operatorname{dim} R^{\prime}=8$, we are done. Consider then $\operatorname{dim} R^{\prime}<8$. Since the projection induces an epimorphism $S \# \mathbf{k} \Gamma \rightarrow$ $R^{\prime} \# \mathbf{k} \Gamma$, we have $\operatorname{dim} R^{\prime}=1,2$ or 4 . Since

$$
\operatorname{dim} R \geq \operatorname{dim} R(0)+\operatorname{dim} R(1)=\operatorname{dim} S(0)+\operatorname{dim} S(1)=1+\operatorname{dim} S(1),
$$

we have $\operatorname{dim} R^{\prime}=2$ or 4 . If $\operatorname{dim} R^{\prime}=2$, then $\operatorname{dim} S(1)=1$ and by the considerations for the rank-1 case, we would have $S=R^{\prime}$. Thus $\operatorname{dim} R^{\prime}=4$. Now 2.7 implies that $\operatorname{dim} R^{\prime}(1)=2$, and then by [AG, Prop 3.1.11], $R^{\prime}(1)$ comes from the abelian case. Furthermore, by the same computations as in 5.0.3 and 5.0.4, the matrix of $R^{\prime}$ is $\left(\begin{array}{rr}-1 & \zeta \\ \zeta & -1\end{array}\right)$ (with $\zeta= \pm 1$ ) in some basis $\{x, y\}$. Now, as in 2.1, we have $x^{2} \in \mathcal{P}(S)$ and $y^{2} \in \mathcal{P}(S)$, and it is straightforward to see that $z=x y-\zeta y x \in \mathcal{P}(S)$. Moreover,

$$
c\left(x^{2} \otimes x^{2}\right)=x^{2} \otimes x^{2}, \quad c\left(y^{2} \otimes y^{2}\right)=y^{2} \otimes y^{2}, \quad c(z \otimes z)=z \otimes z .
$$

Thus, thanks to 2.2 , we have $x^{2}=y^{2}=z=0$. Since $S$ is generated by $x$ and $y$, this implies $\operatorname{dim} S=4$, a contradiction.

\section{REFERENCES}

[AG] N. Andruskiewitsch \& M. Graña, Braided Hopf algebras over non abelian finite groups, Boletín de la Acad. Nac. Cs. (Córdoba) 63 (1999), 45-78. Also in q-alg 9802074.

[AS1] N. Andruskiewitsch \& H.-J. Schneider, Lifting of quantum linear spaces and pointed Hopf algebras of order $p^{3}$, J.Algebra 209 (1998) 659-691.

[AS2] N. Andruskiewitsch \& H.-J. Schneider, Finite quantum groups and Cartan matrices, to appear in Adv.Math.

[AS3] N. Andruskiewitsch \& H.-J. Schneider, Pointed Hopf algebras of dimension $p^{4}$, in preparation.

[Be] M. Beattie An isomorphism theorem for Ore extension Hopf algebras, to appear in Comm. Alg. 
[BDG] M. Beattie, S. Dăscălescu \& L. Grünenfelder, On the number of types of finite-dimensional Hopf algebras, Inventiones Math. 136 1, (1999), 1-7.

[Bu] W. Burnside, Theory of groups of finite order, 2nd edition, Dover Pub., New York, 1955.

[CDR] S. Caenepeel, S. Dăscălescu \& S. Raianu, Classifying pointed Hopf algebras of dimension 16, to appear in Comm. Alg.

[D] S. Dăscălescu Pointed Hopf algebras of dimension $p^{n}$ with large coradical, preprint.

[G] M. Graña, On pointed Hopf algebras of dimension $p^{5}$, to appear in Glasgow Math. Journal.

[Ge] S. Gelaki, On pointed Hopf algebras and Kaplansky's tenth conjecture, J.Algebra 209 (1998), 635-657.

[M] S. Montgomery, Hopf algebras and their actions on rings, AMS (1993), CMBS 82.

[N] W.D. Nichols, Bialgebras of type one, Comm. in Alg. 6 (1978), 1521-1552.

[Ros] M. Rosso, Groupes quantiques et algébres de battage quantiques, CRAS Paris 320 Série I (1995), 145148; Quantum groups and quantum shuffles, Inventiones Math. 133 (1998), 399-416.

[S] M.E. Sweedler, Hopf algebras, Benjamin, New York, 1969.

[Sch] P. Schauenburg, A Characterization of the Borel-like Subalgebras of Quantum Enveloping Algebras, Comm. in Alg. 24 (1996), 2811-2823. 